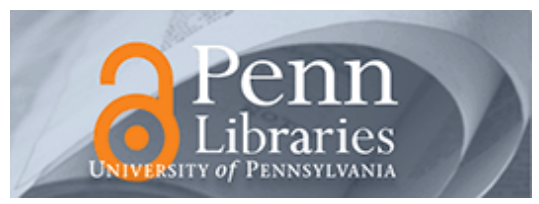

University of Pennsylvania

ScholarlyCommons

6-2007

\title{
Category Management and Coordination in Retail Assortment Planning in the Presence of Basket Shopping Consumers
}

Gerard. P. Cachon

University of Pennsylvania

Gurhan Kok

Follow this and additional works at: https://repository.upenn.edu/oid_papers

Part of the Other Business Commons

\section{Recommended Citation}

Cachon, G. P., \& Kok, G. (2007). Category Management and Coordination in Retail Assortment Planning in the Presence of Basket Shopping Consumers. Management Science, 53 (6), 934-951. http://dx.doi.org/ $10.1287 /$ mnsc. 1060.0661

This paper is posted at ScholarlyCommons. https://repository.upenn.edu/oid_papers/213

For more information, please contact repository@pobox.upenn.edu. 


\title{
Category Management and Coordination in Retail Assortment Planning in the Presence of Basket Shopping Consumers
}

\begin{abstract}
This paper studies the assortment planning problem with multiple merchandise categories and basket shopping consumers (i.e., consumers who desire to purchase from multiple categories). We present a duopoly model in which retailers choose prices and variety level in each category and consumers make their store choice between retail stores and a no-purchase alternative based on their utilities from each category. The common practice of category management (CM) is an example of a decentralized regime for controlling assortment because each category manager is responsible for maximizing his or her assigned category's profit. Alternatively, a retailer can make category decisions across the store with a centralized regime. We show that $\mathrm{CM}$ never finds the optimal solution and provides both less variety and higher prices than optimal. In a numerical study, we demonstrate that profit loss due to CM can be significant. Finally, we propose a decentralized regime that uses basket profits, a new metric, rather than accounting profits. Basket profits are easily evaluated using point-of-sale data, and the proposed method produces near-optimal solutions.
\end{abstract}

\section{Keywords}

game theory, assortment planning, optimization

\section{Disciplines}

Other Business 


\title{
Category Management and Coordination in Retail Assortment Planning in the Presence of Basket Shopping Consumers*
}

\author{
Gérard P. Cachon \\ The Wharton School \\ University of Pennsylvania \\ Philadelphia PA, 19104 \\ cachon@wharton.upenn.edu \\ opim.wharton.upenn.edu/ ${ }^{\sim}$ cachon
}

\author{
A. Gürhan Kök \\ Fuqua School of Business \\ Duke University \\ Durham NC, 27708 \\ gurhan.kok@duke.edu \\ www.fuqua.duke.edu/faculty/alpha/kok.htm
}

July 2004

\begin{abstract}
This paper studies the assortment planning problem with multiple merchandise categories. The categories interact with each other due to the effects of basket shopping consumers. We present a model in which a retailer chooses the number of variants to offer in each category and the consumers of multiple categories (i.e., basket shoppers) make their store choice between the retail store and an exogenous outside alternative based on their total utilities from their shopping basket. We investigate the interaction of the category variety decisions at the retail store under centralized and decentralized management regimes. The common practice of category management is an example of a decentralized regime for controlling assortment because each category manager is responsible for maximizing his or her assigned category's profit. We show that category management never finds the optimal solution and generally provides less variety than optimal. In a numerical study we demonstrate that the profit loss due to category management can be significant. We provide guidelines on which type of categories the retailer should carry and which should have more variety. Finally, we propose a decentralized regime that uses basket profits, a new metric, rather than accounting profits. Basket profits are easily evaluated from available data and this method produces near optimal solutions.
\end{abstract}

${ }^{*}$ The authors would like to thank David Bell, Morris Cohen, Marshall Fisher, Abba Krieger, Debu Prohit, and Ananth Raman for helpful discussions. An electronic copy of this paper is available from the authors' webpages. 
Retailers have increased product selections in all merchandise categories for a number of possible reasons, including heterogeneous customer preferences, variety seeking consumers and brand competition: Quelch and Kenny (1994) report that the number of products in the market place increased by $16 \%$ per year between 1985 and 1992 while shelf space expanded only by $1.5 \%$ per year during the same period. This rapid growth in variety has raised questions as to whether it is excessive. For example, many retailers are adopting an "efficient assortment" strategy, which primarily seeks to find the profit maximizing level of variety by eliminating low-selling products (Kurt Salmon Associates 1993), and "category management", which attempts to maximize the profits within a category (AC Nielsen 1998). There is even empirical evidence that variety levels have become so excessive that reducing variety does not decrease sales (Dreze et al. 1994, Broniarcyzk et al. 1998, Boatwright and Nunes, 2001). And from the perspective of operations within the store and across the supply chain, it is clear that variety is costly: greater variety can lead to slower selling inventory, poor product availability, higher handling costs and greater markdown costs.

While there may be agreement that reducing variety can increase short run profits, there is far less agreement with respect to its impact on long run profits. Assortment planning models in the literature, and organizational forms such as category management, focus on the selection of products in a single category assuming store traffic is exogenous, i.e., variety within a category influences demand conditional on a store visit, but does not influence store choice. However, if, based on single category analyses, a retailer reduces variety in all categories, then the store becomes less attractive and some customers are likely to defect to other retailers, thereby reducing store traffic. This concern is particularly relevant with respect to basket shoppers: if a basket shopper does not find an item that she wants in one category, she may purchase her entire basket from another retailer. Although retailers are well aware of this issue, there is little research on what to do about it.

This paper works with a stylized model to develop managerial insights regarding the assortment planning process in an environment with multiple categories. The retailer offers a selection of products in each merchandise category. Basket shopping consumers choose between the retailer and an outside alternative to maximize their total shopping utility. The retailer's decisions across merchandise categories can be managed under centralized or decentralized regimes. Decentralized regimes (optimization on a category by category basis), such as category management (CM), are analytically manageable but they ignore (in their pure form) the impact of cross-category interac- 
tions. Centralized regimes account for these effects, but only in theory they do so because they are not implementable in practice: it is extremely difficult to design a model to account for all crosscategory effects, to estimate its parameters with available data and to actually solve it. Chen et al (1999) also emphasize the need for models that are solvable with parameters that can be estimated from available data. As CM and centralization are two extremes, merchandising managers often create an intermediate approach by adding constraints to the planning process based on their own knowledge about the store's categories and customers: e.g., include a particular product, have at least two brands in a subgroup, ensure that the timing and depth of promotions are coordinated across obviously complementary products (chips and salsa, beer and pretzels, etc.), etc. But it is not clear if the appropriate constraints are implemented (e.g., should there be at least two brands or five brands, etc.) or whether all of the necessary interactions are accounted for with this ad-hoc approach.

We address the following research questions in this paper. What is the loss due to decentralization compared to the optimal solution? (In some cases decentralization may perform well.) Do decentralized solutions have a consistent bias? (Too much or too little variety.) Is there a way to have the best of both worlds, i.e., are there easily solvable management regimes, based on readily available data, that lead to nearly optimal assortments?

We find that the impact of basket shopping consumers on assortment planning is more complex than one might initially suspect because basket shopping generates two contradictory effects. To illustrate, consider a supermarket example. Capers, a low penetration, low frequency product is often not profitable, but customers that buy capers usually buy large baskets of products. Hence, expanding variety in the spices-condiments category (A) to include capers increases store traffic and sales in some other category (B), i.e., more variety in category A makes adding variety to category B more attractive because category A might pull in more shoppers to the store (i.e., categories are strategic complements). This is an intuitive effect, and it leads to the conclusion that ignoring interactions leads to less than the optimal amount of variety (capers are not included in the assortment if you maximize profit in that category alone; they are included only if total store profit is maximized). However, if category A is very attractive, category B can free ride with lower variety (i.e., categories are strategic substitutes): if the spice-condiment category is deep because of the inclusion of capers, other categories need less variety to attract the same basket shoppers. That effect contradicts the initial intuition, hence, maybe decentralized management 
leads to too much variety. In economic models, activities are usually assumed to be (or found to be) either strategic complements or substitutes, but not both. Our model provides a realistic counter-example.

We characterize the assortment chosen with a decentralized regime, which we refer to as category management (CM), as well as the assortment with a centralized solution (assuming an ideal situation in which we are able to estimate all of the demand parameters, solve the problem, etc.). We show that if there are any basket shoppers, CM never finds the optimal solution and mostly provides less variety than optimal, even though two categories can be either complements or substitutes depending on their variety levels. With numerical examples we demonstrate that the profit loss due to CM can be significant, $28 \%$ on average. More importantly, the performance gets worse as the number of categories increases. Our point is that decentralization can be costly if there are basket shopping consumers and the interactions among categories is not explicitly modeled. To address the potential problem with a decentralized approach to assortment planning, we propose a simple heuristic that retains decentralized decision making (category managers optimize their own categories' profit) but adjusts how profits are measured. To be specific, instead of using an accounting measure of a category's profit, we define a new measure called basket profits. Basket profits can be estimated using point-of-sale data and it enables CM to approximately measure the true marginal benefits of merchandising decisions. We believe this analytical approach is an attractive alternative relative to ad-hoc coordination across category managers.

The next section describes our model, followed by the literature review in Section 2. Section 3 details the consumer choice process and Section 4 presents the solution under different management regimes. Discussion of managerial implications in Section 5 is followed by the heuristic solution in Section 6, a brief numerical study in Section 7 and concluding remarks in Section 8.

\section{Model Basics}

Consider a retailer that carries multiple categories of goods (e.g., shirts, ties, sports jackets). The retailer offers a certain assortment defined by what categories she carries and the selection of products in each category. The set of categories is denoted $J=\{1,2, . ., N\}$ and the set of variants in category $j$ is denoted $S_{j}=\left\{1,2, . ., I_{j}\right\}$. The retailer carries assortment $\mathbf{X}=\left(X_{1}, X_{2}, . ., X_{J}\right)$, where $X_{j} \subset S_{j}$, for all $j$. There is an outside alternative (possibly another retailer) that consumers can choose to do their shopping. The outside alternative carries assortment $\mathbf{Z}=\left(Z_{1}, Z_{2}, . ., Z_{J}\right)$ 
where $Z_{j} \subset S_{j}$, for all $j$. In this paper we take the perspective of the retailer and analyze her decisions $\mathbf{X}$ assuming that $\mathbf{Z}$ is exogenous. It is worth noting here that the outside alternative can be interpreted in three meaningful ways:

I1 the retailer's direct competitor (e.g., the store across the street),

I2 an aggregate representation of the world outside the retailer,

I3 a no purchase option calibrated such that the retailer's share in the model given $X$ and $Z$ is the ratio of its current market share to the maximum potential market share it could achieve by carrying the broadest and deepest possible assortment, $X_{j}=S_{j}$ for all $j \in J$.

We make the following assumptions about the retailer's operations. The cost of providing an assortment $X_{j}$ is $C_{j}\left(X_{j}\right)$, which is increasing, convex in $X_{j}$ and can be parameterized with a scalar $c_{j}$ such that $\partial C_{j}\left(c_{j}\right) / \partial c_{j}>0$ (Appendix A describes a realistic replenishment system that yields convex costs in assortment depth.) Let $p_{j}>0$ be the per-unit margin for all variants in category $j^{1}$. Consistent with the notation for the assortment decisions, a variable name in bold letter denotes a vector of those variables; $\mathbf{c}=\left(c_{1}, \ldots, c_{N}\right)$ and $\mathbf{p}=\left(p_{1}, \ldots, p_{N}\right)$.

There are different types of consumers in the market that are characterized by the contents of their shopping baskets. Let $t$ be the index to the elements of the power set of $J,\{B: B \subseteq J\}$. $B_{t} \subset J$ denotes a shopping basket that contains categories $j \in B_{t}$. The number of type $t$ consumers in the market is randomly distributed with mean $\lambda_{t}$. A consumer of type $t$ purchases exactly one unit of a product from each category in her shopping list $B_{t}$. Consumers have perfect information about the offerings at the retail store and the outside alternative (i.e., $\mathbf{X}$ and $\mathbf{Z}$ ). A type $t$ consumer systematically evaluates the attractiveness of the retailer, $A_{t}(\mathbf{X})$, and the outside alternative, $A_{t}(\mathbf{Z})$, and chooses the alternative that maximizes her total utility from the shopping experience. Rhee and Bell (2002) show that consumers patronize many stores but they typically have a primary affiliation to a "main store" that captures the majority of their spending. The

\footnotetext{
${ }^{1}$ The assumption of identical absolute margins of all variants in a category is restrictive. However, it may be realistic in certain cases. For example, different color/size shirts of the same style have the same price tag. Moreover, Anderson et al. (1992) and Cattani et al. (2003) prove that when customers follow a MNL choice model, optimal pricing policy for a group of variants is an identical absolute mark-up policy. This theoretical result fully supports our assumption.
} 
choice between the retailer and the outside alternative in our model corresponds to the choice of a "main store". Thus, customers in our model do not split their shopping between two stores.

\section{Related Literature}

Interest in category management $(\mathrm{CM})$ is high. According to a recent industry report, $83 \%$ of the grocery retailers view CM as the most important issue facing them (Progressive Grocer 1996). The shift from brand-centered management to $\mathrm{CM}$ resulted in a more profitable pricing structure by eliminating the competitive pricing between brands (AC Nielsen 1998, Basuroy et al. 2001, Zenor 1994). Our paper attempts to shift the discussion from brand level to the category level and raises questions about the interaction among categories.

Chen et al. (1999) also study the impact of basket shopping consumers. They show that merchandising decisions should not be governed by accounting profits, but rather by a new construct they develop called marketing profits. Like us, they argue that simple techniques, based on readily available data, are needed to guide decision making. However, there are some significant differences between their work and ours. In their model each consumer type bases its store choice decision on the variety of a single category, what they call the lead category. Hence, expanding variety in category B has no influence on the store choice decision of category A lead customers. In contrast, our consumers base their decisions on the total utility of their basket, which may include multiple categories. As a result, there are minimal strategic interactions among categories in their model, whereas in our model the strategic interactions are strong and lead to both strategic substitutes and complements. A second key difference is how they improve decision making. They assume a store makes optimal shelf space decisions and then infer marketing profit parameters that would imply those decisions are optimal. They then use those marketing profit estimates to guide other merchandising decisions, such as advertising allocation. We use point of sales data to estimate basket profits, without assuming the retailer's assortment is optimal, and then derive optimal assortment decisions in a decentralized regime.

There are many different modeling approaches to consumer choice over multiple products. See Anderson, de Palma and Thisse (1992) for a review. We follow the approach taken by Guadagni and Little (1983) and others: we presume each consumer receives a random utility from each item in the choice set and the highest utility item is chosen. As a result, increasing the breadth and depth of the assortment in our model increases total demand. The findings in Dhar, Hoch and 
Kumar (2001) are generally consistent with that assumption. However, we recognize that our model does not explicitly account for other possible factors that influence the relationship between assortment variety and demand: the space devoted to a category and the presence or absence of a favorite item influence the perception of variety (Kahn and Lehmann 1991, Broniarczyk et al. 1998) as well as a the arrangement, complexity and presence of repeated items in an assortment (Hoch et al. 1999, Huffman and Kahn 1998, Simonson 1999).

Although research has primarily focused on single category choice decisions, there is recent research that examines multiple category purchases in a single shopping occasion by modeling the dependency across multi-category items explicitly (see Russell et al. 1997 for a review). Manchanda et al. (1999) find that two categories may co-occur in a consumer basket either due to their complementary nature (e.g., cake mix and frosting) or due to coincidence (e.g., similar purchase cycles or other unobserved factors). Bell and Lattin (1998) show that consumers make their store choice based on the total basket utility. Bodapati and Srinivasan (1999) relates feature advertising to store traffic effects using a nested logit framework. In these papers and in ours, consumers first assign a utility to an anticipated market basket and subsequently use this utility to determine store choice.

Fixed costs for each store visit (e.g., search and travel costs) provide an intuitive explanation for why consumers basket shop. Bell, Ho and Tang (1998) use market basket data to analyze consumer store choices and explicitly consider the roles of fixed and variable costs of shopping. We do not explicitly derive optimal baskets - we take them as given.

A number of recent papers study assortment selection and stocking decisions for a group of substitutable products in a single category (e.g., van Ryzin and Mahajan 1999, Mahajan and van Ryzin 2001, Smith and Agrawal 2000, Kok and Fisher 2004) and several papers focus only on stocking decisions (e.g., McGillivray and Silver 1978, Parlar 1988, Netessine and Rudi 2003). Unlike our paper, they assume store traffic is exogenous. Agrawal and Smith (2003) study assortment selection and inventory optimization with consumers that purchase sets of complementary items (e.g., shirt and tie, only shirt, skirt and blouse, etc.). These papers do not consider simple heuristics nor compare their centralized decision making regime to a decentralized regime.

We use game theory to study competitive interactions in our CM (decentralized) regime. Gruca and Sudharshan (1991) and Basuroy and Nguyen (1998) study a market share game based on the Multinomial Logit (MNL) model and demonstrate that certain conditions are needed for equilibrium 
to exist. Although our model of consumers' product and store choice is based on a nested MNL model, our retailer's share equation has the same form as a Multiplicative Competitive Interactions (MCI) model. Karnani (1985) studies an MCI market share model with several firms that compete in a single market through several marketing decisions, including price. Existence of equilibria is not guaranteed in his model, because the profit function of a single firm is not jointly concave in the marketing variables. Our model is similar to his, however we have several customer types corresponding to several markets and we do not include price as a choice variable. Finding the centralized solution in our model with a single customer type corresponds to finding the best response of a firm in Karnani's model and we show that this is not straightforward. Monahan (1987) studies a model in which two firms compete with each other in several markets under an MCI model with a single marketing variable. Our model also has several markets, but the retailer's shares in different markets (customer types) are interdependent and multiple marketing variables (i.e., variety levels in all categories) play a role in each market. Netessine and Zhang (2004) show that decentralized retailers selling strategic complements carry less inventory than optimal. We show that CM provides less variety than optimal in the presence of basket shoppers even though the interactions among categories in our model are not always strategic complements.

\section{Consumer Choice}

Our consumer choice model is based on a nested Multinomial Logit (MNL) framework. A consumer chooses between the retailer and the outside alternative based her expected utility with each option. Her total utility at the retailer (or the outside alternative) is the sum of her utilities from each category in her basket.

In merchandise category $j$, a consumer's utility from purchasing variant $i$ in category $j$ is $u_{j i}=$ $v_{j i}+\varepsilon$ where $\varepsilon$ are $i . i . d$. according to a Gumbel distribution with zero mean and variance $\pi^{2} \mu^{2} / 6$, i.e., $F(y)=\exp \left[-e^{-(y / \mu+\gamma)}\right]$, where $\gamma$ is Euler's constant $(\gamma \approx 0.5722)$. Without loss of generality, we order the variants in a category in decreasing expected utilities, i.e., $v_{j 1} \geq v_{j 2} \geq . . \geq v_{j I_{j}}$. Let $U\left(X_{j}\right)$ be a consumer's utility from the purchase of one variant in category $j$ with assortment $X_{j}$, i.e., $U\left(X_{j}\right)=\max \left\{u_{j i}: i \in X_{j}\right\}$. Because the Gumbel distribution is closed under maximization, $U\left(X_{j}\right)$ follows a Gumbel distributed with mean $E\left[U\left(X_{j}\right)\right]=\mu \ln \sum_{i \in X_{j}} e^{v_{j i} / \mu}$ and scale parameter $\mu$ (Ben-Akiva and Lerman 1985). The analogous results follow for the outside alternative.

Consider a consumer type with a single category basket, i.e., $B_{t}=\{j\}$. From the MNL formula, 
the probability the consumer chooses the retailer over the outside alternative is

$$
s_{t}\left(X_{j}\right)=\frac{\exp \left(E\left[U\left(X_{j}\right)\right] / \mu\right)}{\exp \left(E\left[U\left(X_{j}\right)\right] / \mu\right)+\exp \left(E\left[U\left(Z_{j}\right)\right] / \mu\right)}=\frac{A_{j}\left(X_{j}\right)}{A_{j}\left(X_{j}\right)+A_{j}\left(Z_{j}\right)}, \quad A_{j}\left(X_{j}\right)=\sum_{i \in X_{j}} e^{v_{i} / \mu} .
$$

Now consider a customer with a multi-category basket. Her total basket utility is $L_{t}(\mathbf{X})=$ $\sum_{j \in B_{t}} U\left(X_{j}\right)$, the sum of the utilities of the categories in her basket. $L_{t}(\mathbf{X})$ is not Gumbel because the Gumbel distribution is not closed under addition. The store choice probability of the consumers can not be specified in closed form with the true distribution of $L_{t}$. To be able to use the MNL formula, we show in Appendix B that the distribution of $L_{t}$ can be approximated with a Gumbel distribution. (In particular, the Kolmogorov-Smirnov statistic, the maximum distance between the two distributions, is fairly small across different parameter values.) As for empirical support, Bell and Lattin (1998) use the MNL framework to estimate the basket utilities from consumer basket data and report their model fits the store choice data well.

Let $G_{t}(\mathbf{X})$ be a Gumbel random variable whose mean and variance are the same with $L_{t}(\mathbf{X})$, i.e.,

$$
E\left[G_{t}(\mathbf{X})\right]=\sum_{j \in B_{t}} \mu \ln \sum_{i \in X_{j}} e^{v_{j i} / \mu}=\mu \ln \prod_{j \in B_{t}} \sum_{i \in X_{j}} e^{v_{j i} / \mu}
$$

and $V\left(G_{t}\right)=\left|B_{t}\right| \pi \mu^{2} / 6$. Hence the scale parameter of $G_{t}$ is $\mu \sqrt{\left|B_{t}\right|}$. Consumer type $t$ chooses the maximum of $G_{t}(\mathbf{X})$ and $G_{t}(\mathbf{Z})$ to decide where to shop, and from the MNL share formula

$$
s_{t}(\mathbf{X})=\frac{A_{t}(\mathbf{X})}{A_{t}(\mathbf{X})+A_{t}(\mathbf{Z})}
$$

where

$$
A_{t}(\mathbf{X})=\exp \left(\mu \ln \prod_{j \in B_{t}} \sum_{i \in X_{j}} e^{v_{j i} / \mu} / \mu \sqrt{\left|B_{t}\right|}\right)=\prod_{j \in B_{t}} A_{j}\left(X_{j}\right)^{\frac{1}{\sqrt{\left|B_{t}\right|}}}
$$

Rewrite the share formula:

$$
s_{t}(\mathbf{X})=\frac{\prod_{j \in B_{t}} A_{j}\left(X_{j}\right)^{1 / \sqrt{\left|B_{t}\right|}}}{\prod_{j \in B_{t}} A_{j}\left(X_{j}\right)^{1 / \sqrt{\left|B_{t}\right|}}+\prod_{j \in B_{t}} A_{j}\left(Z_{j}\right)^{1 / \sqrt{\left|B_{t}\right|}}}
$$

The MNL and the Multiplicative Competitive Interactions (MCI) models are presented as alternative formulations in text books and the choice between them depends on the particular application. Our share formula, (3), has the form of a MCI model although our consumer choice model is a nested MNL model. 


\section{Analysis}

In this section, we present a game theoretic analysis of category management (CM), which is our representation of the decentralized regime because each category manager seeks to maximize her own profit. We also characterize the optimal solution for the retailer (i.e., the centralized regime). The analysis of the $\mathrm{CM}$ game is complex because the best response functions are not monotone. Nevertheless, we provide equilibrium existence conditions and we characterize the equilibria. The centralized regime is also not well-behaved, yet we are able to provide some structure and monotonicity results.

The expected profit in category $j$ is

$$
\pi^{j}(\mathbf{X})=p_{j} \sum_{t \mid j \in B_{t}} \lambda_{t} s_{t}(\mathbf{X})-C_{j}\left(X_{j}\right)
$$

Proposition 1 The retailer's optimal assortment in category $j$ is of the type $\left\{0,1,2, . ., x_{j}\right\}$ where $x_{j} \in\left\{1, . ., I_{j}\right\}$.

Proof. The proof is by contradiction. Consider two products $i$ and $k$ where $i<k$. Suppose $k \in X_{j}$ and $i \notin X_{j}$ and let $X_{j}^{\prime}=X_{j} \cup\{i\} \backslash\{k\} \quad$ Since $v_{i} \geq v_{k}, A_{j}\left(X_{j}^{\prime}\right)>A_{j}\left(X_{j}\right)$. We also have $C_{j}\left(X_{j}^{\prime}\right)=C_{j}\left(X_{j}\right)$, because $\left|X_{j}^{\prime}\right|=\left|X_{j}\right|$. Hence $\pi^{j}\left(X_{1}, . ., X_{j}^{\prime}, . . X_{N}\right) \geq \pi^{j}\left(X_{1}, . ., X_{j}, . . X_{N}\right)$.

van Ryzin and Mahajan (1999) prove the same result for a single category by explicitly modeling the inventory costs of each product in a newsvendor framework. As a result, we can think of the variety decision in each category in terms of the number of variants in the category, $x_{j}$ (as opposed to a set of variants). The analogous result holds for the outside alternative, $z_{j}$.

Redefine the attractiveness and share functions with these new decision variables. The variety levels at the retailer and the outside alternative are $\mathbf{x}=\left(x_{1}, x_{2}, . ., x_{J}\right)$, and $\mathbf{z}=\left(z_{1}, z_{2}, . ., z_{J}\right)$ where $x_{j}$ and $z_{j} \in\left\{0,1, . ., I_{j}\right\}$ for all $j . \quad A_{j}:\{0,1,2, ..\} \rightarrow \Re$ and $A_{j}\left(x_{j}\right)=\sum_{i=1}^{x_{j}} e^{v_{j i} / \mu}$. The basket attractiveness function is then $A_{t}(\mathbf{x})=\prod_{j \in B_{t}} A_{j}\left(x_{j}\right)^{1 / \sqrt{B_{t}}}$ and the retailer's share is

$$
s_{t}(\mathbf{x})=\frac{A_{t}(\mathbf{x})}{A_{t}(\mathbf{x})+A_{t}(\mathbf{z})}
$$

Observe that $A_{j}\left(x_{j}\right)$ is nonnegative, increasing and concave in $x_{j}$. Similarly $A_{t}(\mathbf{x})$ is increasing and concave in $x_{j}$ for all $j$ and $t$. Finally, we can rewrite the category profit function.

$$
\pi^{j}(\mathbf{x})=p_{j} \sum_{t \mid j \in B_{t}} \lambda_{t} s_{t}(\mathbf{x})-C_{j}\left(x_{j}\right)
$$


The assortment decision $\mathbf{x}$ is a vector of integers. Hereafter, we work with the continuous version of the problem: differentiability facilitates the derivation and presentation of the results and allows comparative statics; and existence cannot be guaranteed under integrality constraints.

\subsection{Category management}

The common practice of category management (CM) is an example of a decentralized regime for controlling assortment because each category manager is charged with maximizing profit with his or her assigned merchandise category. Since basket shoppers' store choice decision depends on the variety levels of other categories, category $j$ 's optimal variety level depends on the vector of variety levels of the other categories denoted $x_{-j}$ (i.e., $x_{-j}=\left(x_{1}, . ., x_{j-1}, x_{j+1}, . ., x_{N}\right)$. Hence a game theoretic situation arises where category $j$ employs a best response, defined as follows: the strategy $x_{j}\left(x_{-j}\right)$ is category $j$ 's best response to $x_{-j}$ if

$$
x_{j}\left(x_{-j}\right)=\underset{x_{j}}{\arg \max }\left\{\pi^{j}\left(x_{j}, x_{-j}\right), \text { s.t. } 0 \leq x_{j} \leq I_{j}\right\}, \forall j
$$

Category managers do not actually need to know the $\lambda_{t}$ parameters and the definition of the $s_{t}$ functions. We expect them to find the variety level that maximizes the category profits (i.e., the best response function) given other categories' variety levels. They would estimate a demand function, say $d_{j}\left(x_{j}\right)$, as a proxy for $\sum_{t \mid j \in B_{t}} \lambda_{t} s_{t}(\mathbf{x})$ and then solve a single variable concave optimization problem to find $x_{j}\left(x_{-j}\right)$. Note that $d_{j}\left(x_{j}\right)$ depends on the variety levels of other categories.

$\mathrm{CM}$ can be interpreted as an explicit non-cooperative game between the category managers, because each category manager is responsible exclusively for the profits of her own category. Alternatively, it can be interpreted as an iterative application of single category planning where each category's variety level optimized assuming all other assortment decisions for the retailer are fixed. The following theorem establishes the existence of equilibrium and lays the groundwork for further analysis.

Theorem 2 (Existence) A Nash equilibrium in the CM game exists and any Nash equilibrium is characterized by the following conditions.

$$
\sum_{t \mid j \in B_{t}} p_{j} \lambda_{t} \frac{\partial s_{t}(\mathbf{x})}{\partial x_{j}}-C_{j}^{\prime}\left(x_{j}\right)=0, \quad j=1 . . N
$$


Proof. Define $\pi_{x_{j}}^{j}(\mathbf{x}) \equiv \partial \pi^{j}\left(x_{j}, x_{-j}\right) / \partial x_{j}$ and $\pi_{x_{j} x_{k}}^{j}(\mathbf{x}) \equiv \partial^{2} \pi^{j}\left(x_{j}, x_{-j}\right) / \partial x_{j} \partial x_{k}$ :

$$
\begin{aligned}
\pi_{x_{j}}^{j}(\mathbf{x}) & =\sum_{t \mid j \in B_{t}} p_{j} \lambda_{t} \frac{\partial s_{t}(\mathbf{x})}{\partial x_{j}}-C_{j}^{\prime}\left(x_{j}\right) \\
\pi_{x_{j} x_{j}}^{j}(\mathbf{x}) & =\sum_{t \mid j \in B_{t}} p_{j} \lambda_{t} \frac{\partial^{2} s_{t}(\mathbf{x})}{\partial x_{j}^{2}}-C_{j}^{\prime \prime}\left(x_{j}\right)
\end{aligned}
$$

As shown in Appendix $\mathrm{C}, A_{t}(\mathbf{x})$ is concave in $x_{j}$; therefore $s_{t}(\mathbf{x})$ is concave in $x_{j}$, for all $t$. Also $C_{j}^{\prime \prime}\left(x_{j}\right) \geq 0$ by assumption. Consequently, the category profit $\pi^{j}(\mathbf{x})$ is concave in $x_{j}$. Existence of an equilibrium follows from Theorem 1.2. in Fudenberg and Tirole (2000).

Although an equilibrium exists, numerical examples show that there can be multiple equilibria even in the $N=2$ case. Figures 1 and 2 illustrate examples with one and three equilibria respectively. We also found examples with multiple equilibria for $N>2$.

The shape of the reaction functions $x_{j}\left(x_{-j}\right)$ helps us to understand the dynamics of the game from each category's perspective. From (5) and the Implicit Function Theorem,

$$
\frac{\partial x_{j}\left(x_{-j}\right)}{\partial x_{k}}=-\sum_{t \mid\{j, k\} \subseteq B_{t}} p_{j} \lambda_{t} \frac{\partial^{2} s_{t}(\mathbf{x})}{\partial x_{k} \partial x_{j}} / \sum_{t \mid j \in B_{t}} p_{j} \lambda_{t} \frac{\partial^{2} s_{t}(\mathbf{x})}{\partial x_{j}^{2}}-C_{j}^{\prime \prime}\left(x_{j}\right)
$$

The denominator is always negative, therefore the sign of $\partial x_{j}\left(x_{-j}\right) / \partial x_{k}$ depends on the sign of $\partial^{2} s_{t}(\mathbf{x}) / \partial x_{k} \partial x_{j}$ for all $t:\{j, k\} \subseteq B_{t}$. We see from Equation (11) in Appendix C that $\partial^{2} s_{t}(\mathbf{x}) / \partial x_{k} \partial x_{j}<(>) 0$ when $A_{t}(\mathbf{z})>(<) A_{t}(\mathbf{x})$.

Remark 3 i) For each category pair $(j, k), \Re^{N}$ can be divided into three regions: $j$ and $k$ are strategic complements in $X_{j k}^{+}$, substitutes in $X_{j k}^{-}$, and the relation is indeterminate in $\Re^{N}-\left(X_{j k}^{+} \cup\right.$ $\left.X_{j k}^{-}\right)$. ii) Let $X^{+}=\bigcap_{j, k \in J} X_{j k}^{+}$and $X^{-}=\bigcap_{j, k \in J} X_{j k}^{-}$. Clearly $\{\mathbf{x} \leq \mathbf{z}\} \subseteq X^{+}$and $\{\mathbf{x}>\mathbf{z}\} \subseteq X^{-}$.

Proof. i) Define $X_{t}^{+}=\left\{\mathrm{x} \in \Re^{N}, \prod_{j \in B_{t}} A_{j}\left(x_{j}\right)<\prod_{j \in B_{t}} A_{j}\left(z_{j}\right)\right\} . X_{t}^{+}$is the region where the retailer is less attractive to type $t$ consumers than the outside alternative Observe that in $X_{t}^{+}, A_{t}(\mathbf{z})-$ $A_{t}(\mathbf{x})>0$. Let $X_{j k}^{+}=\left\{\mathbf{x} \in X_{t}^{+}, \forall t \mid\{j, k\} \subseteq B_{t}\right\}$. In $X_{j k}^{+}, \pi_{x_{j} x_{k}}^{j}(\mathbf{x})$ is positive, hence $\pi^{j}(\mathbf{x})$ is supermodular in $\left(x_{j}, x_{k}\right)$. Similarly define $X_{t}^{-}=\left\{\mathbf{x} \in \Re^{N}, \prod_{j \in B_{t}} A_{j}\left(x_{j}\right)>\prod_{j \in B_{t}} A_{j}\left(z_{j}\right)\right\}$ and $X_{j k}^{-}=$ $\left\{\mathbf{x} \in X_{t}^{-}, \forall t \mid\{j, k\} \subseteq B_{t}\right\}$. In $X_{j k}^{-}, \pi^{j}(\mathbf{x})$ is submodular in $\left(x_{j}, x_{k}\right)$. Notice that $\Re^{N}-\left(X_{j k}^{+} \cup X_{j k}^{-}\right)$ is not empty, unless there is only one shopping basket with $j$ and $k$ in it. The sign of $\pi_{x_{j} x_{k}}^{j}(\mathbf{x})$ is indeterminate in this region. ii) Note that in vector notation $\{\mathbf{x} \leq \mathbf{z}\}=\left\{\mathbf{x} \mid x_{j} \leq z_{j}\right.$ for all $\left.j\right\}$. $\{\mathbf{x} \leq \mathbf{z}\} \subseteq X_{j k}^{+}, \forall j, k$. Hence $\{\mathbf{x} \leq \mathbf{z}\} \subseteq X^{+}$. Similarly, $\{\mathbf{x}>\mathbf{z}\}=\left\{\mathbf{x} \mid x_{j}>z_{j}\right.$ for all $\left.j\right\} \subseteq X_{j k}^{-}$for all $j, k$. Hence $\{\mathbf{x} \geq \mathbf{z}\} \subseteq X^{-}$. 
In theoretical models with multiple activities, usually one type of interaction (either complements or substitutes) is assumed to hold everywhere or is found to hold everywhere. In our model however, the type of interaction between the categories depends on the attractiveness of all the shopping baskets that contain these two categories, which in turn depends on the variety levels of categories $j, k$, and other categories that co-exist in the shopping baskets with $j$ and $k$. In the $N=2$ case, since there is only one basket type with both $j$ and $k$ in it, we can tell exactly the sign of $\partial x_{j}\left(x_{-j}\right) / \partial x_{k}$ in any region in $\Re^{2}$. The categories are strategic complements if $A_{j}\left(x_{j}\right) A_{k}\left(x_{k}\right)<A_{j}\left(z_{j}\right) A_{k}\left(z_{k}\right)$ and substitutes otherwise. As our analysis suggests, the reaction functions in Figure 1 are increasing-decreasing in the other category's variety level. However, we can not partition the action space as clearly in the $N>2$ case, because we do not know the slope of the best response functions in $\Re^{N}-\left(X_{j k}^{+} \cup X_{j k}^{-}\right)$. Nevertheless, Remark (3) characterizes two distinct regions: In the first region $\left(X^{+}\right)$, all categories are strategic complements, we can say more about the game and its' characteristics by using the theory of supermodular games. In the second region $\left(X^{-}\right)$, all categories are strategic substitutes.

Theorem 4 (Restricted game) Redefine the action space of the category management game (CM) such that each category's profit maximization problem is

$$
\max _{x_{j}}\left\{\pi^{j}\left(x_{j}, x_{-j}\right), \text { s.t. } 0 \leq x_{j} \leq z_{j}\right\}, \forall j
$$

i) This game is supermodular. The equilibrium set is non-empty and has a largest and a smallest element. The largest element is the Pareto best and the smallest element is the Pareto worst equilibrium. The largest and the smallest equilibria are increasing in $\mathbf{p}$ and $\boldsymbol{\lambda}$ and decreasing in $\mathbf{z}$ and $\mathbf{c}$. ii) In a symmetric game where data for all categories are identical, there exists only symmetric equilibria.

Proof. $i$ ) The feasible action space $\{\mathbf{0} \leq \mathbf{x} \leq \mathbf{z}\}$ is a non-empty, convex and compact set. The payoff function $\pi^{j}(\mathbf{x})$ is supermodular in all pairs of variables in $\{\mathbf{x} \leq \mathbf{z}\} \subseteq X^{+}$from Remark (3). Supermodularity of $\pi^{j}$ in $\left(x_{j}, x_{k}\right)$ for all $j, k$ implies that $\pi^{j}\left(x_{j}, x_{-j}\right)$ has increasing differences in $\{\mathbf{0} \leq \mathbf{x} \leq \mathbf{z}\}$. Therefore $\left(C M_{-} R\right)$ is a supermodular game. The payoff function $\pi^{j}$ is continuous in $x_{j}$ for each $x_{-j}$ in $\{\mathbf{0} \leq \mathbf{x} \leq \mathbf{z}\}$. Then by Theorem 4.2.1. of Topkis (1998), the set of equilibrium points is a non-empty complete lattice and a greatest and a least equilibrium point exist. Since the payoff to a player is increasing in other players' strategies, i.e., $\frac{\partial \pi^{j}(\mathbf{x})}{\partial x_{k}} \geq 0$ for all $k \neq j$, the largest element is the Pareto best and the smallest element is the Pareto worst equilibrium. This 
result follows from a simple step-wise improvement argument, see Section 2.2.3. of Vives (1999). We parameterize the game $\left(C M_{-} R\right)$ by $(\mathbf{p}, \boldsymbol{\lambda},-\mathbf{z},-\mathbf{c})$. It is easy to show that $\pi^{j}$ is continuous in $x_{-j}$ for every $(\mathbf{p}, \boldsymbol{\lambda},-\mathbf{z},-\mathbf{c})$ and it has increasing differences in $x_{j}$ and $\mathbf{p}, \boldsymbol{\lambda},-\mathbf{z}$, and $-\mathbf{c}$. By Theorem 4.2.2. of Topkis (1998) we obtain the monotonicity results.

ii) The proof is by contradiction. Let $\mathbf{x}$ be an asymmetric equilibrium with $x_{j}>x_{k}$ for some $j$ and $k$. By definition of equilibrium $\mathbf{x}$ satisfies (5) for all categories including $j$ and $k$ :

$$
\sum_{t \mid j \in B_{t}} p_{j} \lambda_{t} \frac{\partial s_{t}(\mathbf{x})}{\partial x_{j}}-C_{j}^{\prime}\left(x_{j}\right)=0 \text { and } \sum_{t \mid j \in B_{t}} p_{k} \lambda_{t} \frac{\partial s_{t}(\mathbf{x})}{\partial x_{k}}-C_{k}^{\prime}\left(x_{k}\right)=0 .
$$

Now,

$$
\frac{\partial s_{t}(\mathbf{x})}{\partial x_{j}}=\frac{\partial s_{t}\left(x_{j}, x_{-j}\right)}{\partial x_{j}}<\frac{\partial s_{t}\left(x_{k}, x_{-j}\right)}{\partial x_{k}}<\frac{\partial s_{t}\left(x_{k}, x_{-k}\right)}{\partial x_{k}}=\frac{\partial s_{t}(\mathbf{x})}{\partial x_{j}}
$$

The first inequality follows from the concavity of $s_{t}$ in $x_{j}$ and $x_{j}>x_{k}$. The second inequality follows from the supermodularity of $s_{t}()$ and $x_{-j}<x_{-k}$. Also we have $p_{j}=p_{k}$ and $C_{j}^{\prime}\left(x_{j}\right) \geq C_{k}^{\prime}\left(x_{k}\right)$. Hence the LHS of the equation for $j$ is always strictly smaller than the one for $k$. As a result, both conditions can not be satisfied at the same time and $\mathbf{x}$ can not be an equilibrium.

The action space defined in Theorem 4 is quite realistic under interpretation (I2) of the outside alternative, because $\mathbf{x}<\mathbf{z}$ implies that the retailer's market share is less than half of the whole market. Even in the original game with the unrestricted strategy space, if the cost of providing variety is too high and all the equilibria are in $X^{+}$, the results of Theorem 4 apply.

In a symmetric game, the categories have the same price, cost, competition, and demand vectors. Second part of the above Theorem rules out the asymmetric equilibria in a symmetric game (CM_R). The same result can also be established for the submodular region of the game (see part 3 of Proposition 11 in Appendix D), but we are only able to rule out asymmetric equilibria for the whole game in the case of two symmetric categories.

Theorem 5 In the game $C M$ with $N=2$ and two categories are symmetric, there exists only symmetric equilibria.

Proof. i) By the second part of Theorem (4) and part (iii) of Proposition (11), the only region that can have asymmetric equilibria is $\Re^{2}-\{\mathbf{x} \leq \mathbf{z}\}-X^{-}$. This region is a subset of $X^{+}$and it is composed of two distinct parts on each side of the diagonal, denoted $F_{1}$ and $F_{2}$ (see Figure 3 for illustration of these regions. Reaction functions are symmetric. If $x_{1}\left(x_{2}\right)$ passes through one of 
$F_{1}$ or $F_{2}, x_{2}\left(x_{1}\right)$ passes through the other and vice versa. Hence there cannot be an intersection in either $F_{1}$ or $F_{2}$.

\subsection{Optimal solution}

In the centralized system, total store profits are optimized over all category variety levels.

$$
\max _{\mathbf{x}}\left\{\Pi(\mathbf{x})=\sum_{j=1}^{N} \pi^{j}(\mathbf{x}), \text { s.t. } 0 \leq x_{j} \leq I_{j}, \forall j\right\}
$$

Differentiate $\Pi(\mathbf{x})$ with respect to $x_{j}$.

$$
\frac{\partial \Pi(\mathbf{x})}{\partial x_{j}}=\sum_{t \mid j \in B_{t}} p_{j} \lambda_{t} \frac{\partial s_{t}(\mathbf{x})}{\partial x_{j}}-C_{j}^{\prime}\left(x_{j}\right)+\sum_{\substack{k=1 \\ k \neq j}}^{N} \sum_{t \mid j, k \in B_{t}} p_{k} \lambda_{t} \frac{\partial s_{t}(\mathbf{x})}{\partial x_{j}}
$$

The marginal effect of $x_{j}$ on the total profit is composed of its impact on own and cross category sales. After reorganizing terms, the first order conditions for optimality are

$$
\sum_{t \mid j \in B_{t}}\left(\sum_{k \in B_{t}} p_{k}\right) \lambda_{t} \frac{\partial s_{t}(\mathbf{x})}{\partial x_{j}}-C_{j}^{\prime}\left(x_{j}\right)=0, \forall j
$$

The first order optimality conditions are based on the total profit earned from each customer type, whereas the CM solution is based on the category profits. Since $s_{t}(\mathbf{x})$ is concave in $x_{j}$, $\partial^{2} \Pi(\mathbf{x}) / \partial x_{j}^{2}<0$, for all $j$ and $t$, and the first order condition (9) characterize the unique optimal variety level $x_{j}$ for fixed $x_{-j}$. However, we are not able to show that the joint optimization problem is unimodular, therefore the system of equations $\partial \Pi(\mathbf{x}) / \partial x_{j}=0$ for $j=1, . ., N$ is not sufficient for optimality. It is easy to find numerical examples where $\Pi(\mathbf{x})$ is not jointly concave, even with $N=2$.

We start the characterization of the optimal solution by describing two special cases of problems. Note that Case A problems include all two category problems including the Case B problems.

Case A $N$ categories. Basket types have the following property: $\left|\left\{B_{t}: j, k, l \in B_{t}\right\}\right| \leq 1$ for all $j, k, l$ such that $j \neq k, j \neq l$, and $k \neq l$.

Case B $N=2$. Symmetric categories. All customers are basket shoppers (i.e., $\lambda_{t}>0$ only for $\left.B_{t}=\{1,2\}\right)$. The products in a category are equally popular (i.e., $A_{j}(x)$ is linear). Cost of variety is linear (i.e., $\left.C_{j}^{\prime \prime}(x)=0\right)$. 
Theorem 6 Consider the special case A. i) If a symmetric solution to the first order conditions of OPT (9) satisfies $A_{t}(\mathbf{x})>\frac{2 \tau-1}{2 \tau+1} A_{t}(\mathbf{z})$ where $\tau=1 / \sqrt{\left|B_{t}\right|}$ for any $t$, it is a local optimal solution and can not be part of a continuum of local optima. ii) There exist at most one symmetric solution to (9) in $X^{-}$.

Proof. i) A sufficient condition for a stationary point to be a local maximum is that the Jacobian of $\Pi_{x_{j}}^{j}=0, j=1 . . N$ is diagonally dominant, because a symmetric diagonally dominant matrix with negative diagonal entries is negative definite (Corollary 7.2.3 of Horn and Johnson, 1985). We need $\Pi_{x_{j} x_{j}}^{j}(\mathbf{x})+\sum_{k \neq j}\left|\Pi_{x_{j} x_{k}}^{j}(\mathbf{x})\right|<0$ and $\mathbf{x} \in X$. Replace $p_{j}$ in the proof of Proposition 11 with $\sum_{l \in B_{t}} p_{l}$ and show that diagonal dominance is achieved if $A_{t}(\mathbf{x})>\frac{2 \tau-1}{2 \tau+1} A_{t}(\mathbf{z})$ is satisfied. Hence, in this interval $\Pi(\mathbf{x})$ is jointly concave at the symmetric stationary points. $i i)$ Following the same line of analysis we did for the game $(C M)$, we can show that $\frac{\partial \Pi(\mathbf{x})}{\partial x_{k} \partial x_{j}}<0$ in $X^{-}$. Therefore, the solution to the first order condition for category $j$ for fixed $x_{-j}$ is decreasing in $x_{-j}$ in $X^{-}$. Decreasing reaction functions can intersect at the diagonal at most once.

Theorem 7 Consider the special case B. Focus only on the symmetric solutions $\mathbf{x}=(x, x)$. The profit function is convex-concave in $x$, therefore either $(0,0)$ or the largest stationary point with positive profit is the global optimal solution.

Proof. Total profit function is $\Pi(x, x)=\left(p_{1}+p_{2}\right) x^{2 \tau} /\left(x^{2 \tau}+z^{2 \tau}\right)-C_{1}(x)-C_{2}(x)$. The second derivative is given by

$$
\frac{\left(p_{1}+p_{2}\right) 2 \tau x^{2 \tau-2} z^{2 \tau}}{\left(x^{2 \tau}+z^{2 \tau}\right)^{3}}\left(x^{2 \tau}(-1-\tau)+z^{\tau}(\tau-1)\right)
$$

whose sign is positive at $x=0$, decreases with $x$, and is negative for large values of $x$. Hence the profit function is convex-concave in $x$. The function is decreasing for large $x$, therefore there is a local maximum in the concave part of the function, which is the global optimum if it yields positive profit.

Similar to the decentralized solution, the theory of supermodularity enables us to characterize the properties of the optimal solution and provide comparative statics for the restricted problem.

Theorem 8 (Restricted problem) Redefine the action space of the optimization problem (OPT) as

$$
\max _{\mathbf{x}}\left\{\Pi(\mathbf{x})=\sum_{j=1}^{N} \pi^{j}(\mathbf{x}), \text { s.t. } 0 \leq x_{j} \leq z_{j}, \forall j\right\}
$$


i) The set of solutions to the first order conditions (9) of OPT_R has a smallest and a largest element that are monotone increasing in $\mathbf{p}$ and $\boldsymbol{\lambda}$ and decreasing in $\mathbf{z}$ and $\mathbf{c}$. ii) The set of optimal points $\arg \max \left\{\Pi(\mathbf{x})\right.$, s.t., $\left.0 \leq x_{j} \leq z_{j}, \forall j\right\}$ is non-empty, has a smallest and a largest element that are monotone increasing in $\mathbf{p}$ and $\boldsymbol{\lambda}$ and decreasing in $\mathbf{z}$ and $\mathbf{c}$.

Proof. $i$ ) The existence of a largest and a smallest equilibrium and the monotonicity of the set in the parameters follow from applying the theory of supermodular games to the first order optimality conditions, similar to the proof of Theorem 4. However, a stationary point may not be a local maximum. $i$ ) The result on the set of optimal points follow from the optimization of a supermodular function (Theorem 2.8.3 in Topkis, 1999).

\subsection{Comparison of the centralized and decentralized solutions}

Let $X^{c m}$ be the set of Nash equilibria in the $C M$ game. Similarly $X^{o}$ is the set of stationary points of the optimal profit function, OPT. We assume the optimal solution is interior. The following theorems show that $C M$ never finds the optimal solution as long as there are basket shoppers.

Theorem 9 Suppose that there exists at least one consumer type with more than one category in their basket and positive demand rate, i.e., $\left|B_{t}\right|>1$ and $\lambda_{t}>0$. i) The set of Nash equilibria $X^{c m}$ in the category management game, CM, does not contain the global optimal solution to OPT. $X^{\mathrm{cm}}$ does not contain any of the local optimal solutions either. ii) If data is symmetric across all categories, then there is a symmetric stationary point in $X^{o}$ greater than any symmetric equilibrium in $X^{c m}$ (and it is a local optimum if it satisfies $A_{t}(\mathbf{x})>\frac{2 \tau-1}{2 \tau+1} A_{t}(\mathbf{z})$ ). iii) Consider the special case B. The optimal solution is greater than the largest symmetric equilibrium.

Proof. Compare the first order conditions to the optimization problem $(O P T)$ given by $(9)$ and the game $(C M)$ given by $(6)$ :

$$
\Pi_{x_{j}}(\mathbf{x})-\pi_{x_{j}}^{j}(\mathbf{x})=\sum_{k=1, k \neq j}^{N} \sum_{t \mid j, k \in B_{t}} p_{k} \lambda_{t} \frac{\partial s_{t}(\mathbf{x})}{\partial x_{j}}>0
$$

if there is one basket type with more than one category and positive demand rate. Therefore, no point $\mathbf{x}$ can satisfy both set of conditions, i.e., $X^{c m} \cap X^{o}=\emptyset$. ii) For fixed vector of variety levels of other categories, $x_{-j}$, the solution to the first order conditions for category $j\left(x_{j}^{o}\left(x_{-j}\right)\right)$ is always larger than the best response of category $j$ in the game $\left(x_{j}^{c m}\left(x_{-j}\right)\right)$. In the symmetric game, let the 
largest symmetric equilibrium have variety level $x^{c m}$ for all categories. It is sufficient to consider an arbitrary category. We have $x_{j}^{o}\left(x_{-j}^{c m}\right)>x_{j}^{c m}\left(x_{-j}^{c m}\right)=x^{c m}$. We also know that $x_{j}^{o}\left(x_{-j}^{c m}\right)$ does not go to infinity since it is decreasing in $X^{-}$. Therefore, $x_{j}^{o}\left(x_{-j}^{c m}\right)$ will cross the 45 degree line at least once more and the intersection is a stationary point of $(O P T)$, i.e., $\exists x^{1}: x_{j}^{o}\left(x_{-j}^{1}\right)=x^{1}>x^{c m}$. iii) Follows from part $(i i)$ and that the largest stationary point is the optimal solution in case B according to Theorem 7 .

Theorem 10 (Restricted Problem) Suppose that there exists at least one consumer type with more than one category in their basket and positive demand rate, i.e., $\left|B_{t}\right|>1$ and $\lambda_{t}>0$. The global optimal solution of the optimization problem $O P T_{-} R, \mathbf{x}^{o}$, has strictly higher variety in at least one category than any of the Nash equilibria of the game $C M_{-} R$, including the Pareto best equilibrium, i.e., $\exists j, x_{j}^{o}>x_{j}^{c m}, \forall \mathbf{x}^{c m} \in X^{c m}$.

Proof. Let $\mathbf{x}^{o}$ be the optimal solution to $\left(O P T_{-} R\right)$ and $\mathbf{x}^{c m}$ be any equilibrium of the game $\left(C M_{-} R\right)$. By contradiction, suppose that $\mathbf{x}^{o}<\mathbf{x}^{c m}$.

$$
\Pi\left(\mathbf{x}^{o}\right)=\sum_{j} \pi^{j}\left(x_{j}^{o}, x_{-j}^{o}\right)<\sum_{j} \pi^{j}\left(x_{j}^{o}, x_{-j}^{c m}\right)<\sum_{j} \pi^{j}\left(x_{j}^{c m}, x_{-j}^{c m}\right)=\Pi\left(\mathbf{x}^{c m}\right)
$$

This is a contradiction with the optimality of $\mathbf{x}^{o}$. The first inequality follows from $\partial \pi^{j}(\mathbf{x}) / \partial x_{k} \geq 0$ for all $k \neq j$ and $\mathbf{x}$, and the second inequality follows from the definition of the equilibrium, i.e., $x_{j}^{c m}=\arg \max _{x_{j}} \pi^{j}\left(x_{j}, x_{-j}^{c m}\right)$.

Note that CM and OPT would yield the same solution if all customers were single category shoppers. Also, due to the supermodularity of the profit functions in the restricted strategy space, discrete versions of Theorems 4, 8 and 10 are easily established with the following slight modification in Theorem 10: the optimal solution provides greater than or equal to (not strictly greater than) variety than the equilibrium solution in at least one category.

Figures 2 and 4 present pathological examples demonstrating some potential outcomes. Figure 2 shows that even the best of three CM equilibria provides significantly less variety than the optimal solution. In Figure 4 the unique CM equilibrium has the retailer eliminating both categories although the optimal solution has positive variety in both categories. 


\section{Properties of the Optimal and Equilibrium Assortments}

In this section, we investigate the effect of perturbations on the optimal and equilibrium solutions and make inferences about how to allocate resources to categories. We assume the optimal solution, $\mathbf{x}^{c}$, and the equilibria $\mathbf{x}^{d}$ are an interior, so they are responsive to small parameter changes. We classify categories into two types: A basket category has a high co-occurrence rate in baskets with other categories; an independent category has most of its demand from single category shoppers.

\subsection{Best collection of categories}

Consider the scenario with $N$ symmetric categories but focus on two categories $j$ and $k$. Let subscripts $j$ and $k$ denote the individual category shoppers of the respective categories. Also $j k$ denotes the consumers with shopping basket $\{j, k\}$. Given a variety level, the retailer's share of customers with individual categories is larger than its share of basket shoppers if and only if the retailer provides less variety than the outside alternative: $s_{j}>s_{j k}$ and $s_{k}>s_{j k}$ if and only if $\mathbf{x}^{o}<\mathbf{z}$. Hence, the retailer prefers to have a higher proportion of basket shoppers among its customers of categories $j$ and $k$ when $\mathbf{x}^{o}>\mathbf{z}$ (when its market share is greater than $50 \%$ of its potential). This occurs because it is easier for a larger retailer to attract basket shoppers than a small retailer (basket shoppers choose based on multiple categories rather than one). The result implies that a retailer is better off with a collection of independent categories if $\mathbf{x}^{o}<\mathbf{z}$ and better off with basket categories if $\mathbf{x}^{o}>\mathbf{z}$. The situation a retailer is in probably depends on how the outside alternative is interpreted. With interpretation I2, the outside alternative is the aggregate representation of all the other firms, so the retailer probably has less than $50 \%$ of the whole market and is better off with a collection of independent categories. With interpretation I3, the retailer probably has more than $50 \%$ of its market potential, so the retailer prefers to carry basket categories. I3 is the better interpretation if a retailer's demand would increase by less than $100 \%$ if it were to carry a full assortment in every category.

\subsection{Which categories should have more variety?}

Consider two categories with same total demand but one category is more of a basket category than the other. In which category should the retailer provide more variety? Let subscripts $j, k$, and $l$ denote the individual category shoppers of the respective categories. Also $j k$ and $j l$ denote the consumers with shopping baskets $\{j, k\}$ and $\{j, l\}$, respectively. Suppose the demand rate of 
all baskets with variant $k$ are identical to those with $l$. The only exception is the rate of individual category shoppers $\left(\lambda_{k}\right.$ and $\left.\lambda_{l}\right)$ and basket shoppers of $j$ and $k$ or $j$ and $l\left(\lambda_{j k}\right.$ and $\left.\lambda_{k l}\right)$. Let $\lambda_{k}=\Lambda+\delta, \lambda_{j l}=\Lambda-\delta, \lambda_{l}=\Lambda-\delta, \lambda_{j l}=\Lambda+\delta$ where $\Lambda \geq \delta \geq 0$. If $\delta=0$, categories $k$ and $l$ are identical, as $\delta$ increases, demand to each category remains constant, $l$ becomes more of a basket category.

By the implicit function theorem, $\partial x_{k}^{c} / \partial \delta$ has the same sign as

$$
\left.\frac{\partial^{2} \Pi(\mathbf{x})}{\partial \delta \partial x_{j}}\right|_{\mathbf{x}^{o}}=p_{k} s_{k}^{\prime}\left(x_{k}^{o}\right)-\left(p_{j}+p_{k}\right) \frac{\partial s_{j k}\left(x_{j}^{o}, x_{k}^{o}\right)}{\partial x_{k}}
$$

Similarly, $x_{l}^{c}$ increase with $\delta$ if

$$
p_{l} s_{l}^{\prime}\left(x_{l}^{c}\right)<\left(p_{j}+p_{l}\right) \frac{\partial s_{j l}\left(x_{j}^{o}, x_{l}^{o}\right)}{\partial x_{l}}
$$

If categories $k$ and $l$ are symmetric, then as $\delta$ increases from zero, $x_{k}^{o}$ and $x_{l}^{o}$ move in reverse directions. In $\S 4.1$, we showed that $\partial^{2} s_{j l}\left(x_{j}, x_{l}\right) / \partial x_{j} \partial x_{l}>0$ if $A_{j}\left(x_{j}\right) A_{l}\left(x_{l}\right)<A_{j}\left(z_{j}\right) A_{l}\left(z_{l}\right)$ and $>0$ otherwise. Hence $\partial s_{j l}\left(x_{j}, x_{l}\right) / \partial x_{l}$ is an increasing-decreasing function of $x_{j}$. There may be an interval of $x_{j}^{o}$ say, $\left(\underline{x}_{j}^{o}, \bar{x}_{j}^{o}\right)$, where the inequality is satisfied and $\partial x_{l}^{o} / \partial \delta>0$, i.e. the optimal variety level of the basket category is higher than the optimal variety of the independent category. For $x_{j}^{o}<\underline{x}_{j}^{o}$ and $x_{j}^{o}>\bar{x}_{j}^{o}$, the reverse is true. The intuition for this result is as following. If $x_{j}$ is too low, it is not possible to attract many basket shoppers by increasing the variety of the basket category only, so it is better to increase variety in the independent category. If $x_{j}$ is in that interval, the basket category should be assigned more variety. As $x_{j}$ gets too large, there is no need to increase variety in the basket category $l$ because $j$ 's variety level is already attracting many basket customers to category $l$. To illustrate this result, we generated a series of numerical examples by varying $c_{j}$ to control $x_{j}^{c}$.

Similar analysis yields that the equilibrium variety levels display the same properties. $x^{c m}$ increases with $\delta$ when $x_{j}^{c m} \in\left(\underline{x}_{j}^{c m}, \bar{x}_{j}^{c m}\right)$ which is defined by the region $p_{l} s_{l}^{\prime}\left(x_{l}^{c m}\right)<p_{l} \partial s_{j l}\left(x_{j}^{c m}, x_{l}^{c m}\right) / \partial x_{l}$. With symmetric categories $p_{l}<p_{j}+p_{k}$, hence $\left(\underline{x}_{j}^{c m}, \bar{x}_{j}^{c m}\right) \subset\left(\underline{x}_{j}^{c m}, \bar{x}_{j}^{c m}\right)$, i.e., there may be cases where the optimal solution assigns more variety to the basket category and the category management solution does the reverse.

\section{Category management with basket profits}

The CM equilibrium solution emerges as the outcome of a natural iterative process: category managers set variety levels, store traffic and sales are realized, category managers reassess the 
demand function for their category and choose new assortments, etc. The same process occurs if the retailer does category by category optimization. Either way, the process converges to one of the CM equilibria. Despite its simplicity, we show in the next section that CM can significantly deviate from the optimal solution. However, it is not easy to implement the centralized optimal solution: that solution involves estimating the number of customers for all $2^{N}$ basket types and an $N$-dimensional optimization of an ill-behaved function.

In this section we introduce a decentralized heuristic that promises the best of both worlds (i.e., the simplicity of CM and a profit level close to that of the optimal solution). The main weakness of $\mathrm{CM}$ is that it fails to recognize the intercategory effects of variety decisions and underestimates the marginal benefit of variety. As we have seen, the marginal benefit of variety is quite complex. Consider basket shoppers that purchases from two categories, $A$ and $B$. Increasing variety in $A$ increases demand from these shoppers in both category $A$ and $B$. But more variety in $A$ may also warrant a change in the variety of $B$, depending on the current variety levels: variety should be added to $B$ if they are acting as complements and less if they are substitutes. From the perspective of the manager of category $A$, an additional sale is only worth $p_{A}$, but from the retailer's perspective it is worth more. We approximate the true marginal benefit to the retailer with the weighted average of the gross margins across basket types. We call this new metric basket profits because it measures the total profit earned from a customer. Specifically, let $\hat{p}_{j}$ be the basket profit from category $j$ :

$$
\hat{p}_{j}=\sum_{t \mid j \in B_{t}}\left(\lambda_{t} \sum_{l \in B_{t}} p_{l}\right) / \sum_{t \mid j \in B_{t}} \lambda_{t}
$$

The manager for category $j$ is then measured with the following profit function:

$$
\hat{\pi}^{j}(\mathbf{x})=\hat{p}_{j} \sum_{t \mid j \in B_{t}} \lambda_{t} s_{t}(\mathbf{x})-C_{j}\left(x_{j}\right)
$$

Each CM then chooses variety

$$
x_{j}^{B}\left(x_{-j}\right)=\underset{x_{j}}{\arg \max }\left\{\hat{\pi}^{j}\left(x_{j}, x_{-j}\right) \text {, s.t. } 0 \leq x_{j} \leq I_{j}\right\}, \forall j .
$$

Note that all our results on the CM equilibria apply directly to the CM_B heuristic. If we compare the derivative of the CM's profit function to the optimality conditions (9), we see that CM_B uses a weighted average $\hat{p}_{j}$ for all consumer types instead of using $\sum_{k \in B_{t}} p_{k}$ for consumer type $t$. This approximation should work well if $\partial s_{t}(\mathbf{x}) / \partial x_{j}$ for all $t$ are close to each other, however, 
even in symmetric category examples, $\partial s_{t}(\mathbf{x}) / \partial \mathbf{x}_{j}$ varies with the basket size. From a practical perspective, note that the basket profit for each category is easily computed from POS data: it is the average gross margin earned from customers who purchased a basket including that category.

\section{$7 \quad$ Numerical Results}

This section reports on numerical study that focuses on the magnitude of the profit loss due to CM relative to the optimal solution and the performance of $\mathrm{CM}$ with basket profits.

Group 1: We generated 72 symmetric scenarios from all combinations of the following parameters:

$$
\begin{array}{lll}
p=1 & z_{j}=\{5,10\} & C_{j}(x)=c_{j} x \\
A_{j}(x)=x & N=\{2,3,5\} & c_{j}=\{2,4\}
\end{array}
$$

Demand in each category is 100 units and demand is divided across baskets according to the basket ratio vectors given in Table 1 . For example, the vector $\{.2, .6, .2\}$ for $N=3$ means that $20 \%$ of the demand goes to baskets of size one, $60 \%$ goes to baskets of size two, and $20 \%$ to baskets of size three. For category 1, there are two baskets of size two, $\{1,2\}$ and $\{1,3\}$ and 60 units of demand is equally divided among them. Hence, $\lambda_{1}=20, \lambda_{2}=20, \lambda_{3}=20, \lambda_{12}=30, \lambda_{13}=30, \lambda_{23}=30$, and $\lambda_{123}=20$.

The unique CM equilibrium has no variety in 12 out of the 72 scenarios, resulting in a $100 \%$ profit loss relative to the optimal solution. In 21 scenarios there are multiple CM equilibria, one of which was the zero equilibrium. In those cases we assumed the better equilibrium prevails. Table 2 presents summary data on the performance of CM: the average profit loss due to decentralization is $28 \%$ and on average variety is $44 \%$ less than optimal. The performance of CM deteriorates with more categories $(N)$, higher outside variety $(z)$, higher variety costs $(c)$ and a higher proportion of basket shoppers.

Group 2: To explore asymmetric categories, we focus on the $N=2$ cases of Group 1. A total of 48 examples are generated from all combinations of $z_{j} \in\{5,10\}$ and $c_{j} \in\{2,4\}$ for the two categories, i.e., 16 examples for each of the three basket types. The average CM loss is $8 \%$ in the asymmetric examples in comparison to the $13 \%$ loss for the two symmetric category examples in Group 1.

Table 3 presents results for CM with basket profits (CM_B) in comparison to traditional CM and the optimal solution for the examples with $N=2$ from both Groups 1 and 2 and with $N=3$ from Group 1. Like CM, CM_B may lead to multiple equilibria. We first consider the best 
equilibrium of CM_B. The maximum loss with basket profits is only $2.1 \%$ and the average loss is only $0.2 \%$. Variety with basket profits is on average $4.6 \%$ higher than optimal. Furthermore, CM_B works equally well for asymmetric and symmetric scenarios. One drawback of the basket profits heuristic is that it could converge to a bad equilibrium in case of multiple equilibria. Table 3 shows that even the worst equilibrium of CM_B performs better than the best equilibrium of CM (i.e., average profit loss for worst of CM_B is $8.5 \%$ whereas it is $13.2 \%$ for best of CM). The reason for inferior performance is that all categories have zero variety in the worst equilibria in our experiments (CM_B usually has a unique equilibrium in asymmetric examples, and as a result performs well). Starting with a high variety level when a category is first introduced ensures that CM_B (or CM) converges to the best equilibrium.

\section{Conclusion}

We study a retailer's assortment planning problem with multiple categories and basket shopping consumers that choose between the retailer and an outside alternative. We investigate the retailer's assortment decisions across categories under centralized and decentralized management regimes. We find that variety levels between categories can be either strategic complements or strategic substitutes. Nevertheless, we also find that decentralized assortment planning, as with category managers responsible for their own category's profit, is likely to lead to lower than optimal variety and significantly lower profits than optimal. But a centralized optimal solution is almost surely not implementable in practice due to the complexity of the required data estimation and optimization. Therefore, we propose a decentralized regime, like category management, but instead of evaluating each category manager's accounting profit, we measure their basket profits, where basket profits are estimated using point of sales data. We find that our basket profit approach provides nearly the optimal variety and optimal profit. Hence, although the presence of basket shopping consumers is known to create significant analytical complications for the assortment planning problem, a robust and simple analytical solution exists.

\section{A Example of a replenishment system with convex operating cost}

Consider a single category with $x$ products and demand $\lambda(x)=A(x)(A(x)+A(z))^{-1}$ where $A^{\prime}(x)>0$ and $A^{\prime \prime}(x) \leq 0$. Item $i$ 's demand rate is $\lambda_{i}(x)$, where $\sum_{i} \lambda_{i}(x)=\lambda(x)$. Total shelf space capacity is $S$ and assume each item's maximum inventory level is proportional to its demand 
rate, $S_{i}=S \lambda_{i}(x) / \lambda(x)$. There are commercial algorithms that allocate shelf space in this way (see Bultez and Naert 1988 for references). In a continuous review inventory system with zero lead time an order is placed whenever the inventory level is zero and the average inventory level for a single product is $S_{i} / 2$. Total average inventory is $S / 2$, hence inventory holding cost is independent of $x$. The number of orders per unit time for item $i$ is $\lambda_{i}(x) / S_{i}(x)=\lambda(x) / S$, i.e., the order frequency is identical across all items. The total number of orders in the category is $x \lambda(x) / S$, whose second derivative is

$$
\begin{aligned}
\frac{2 \lambda^{\prime}(x)+x \lambda^{\prime \prime}(x)}{S} & =\frac{A(z)}{(A(x)+A(z))^{3}}\left(2 A ^ { \prime } ( x ) \left(\left(A(x)+A(z)-x A^{\prime}(x)+A^{\prime \prime}(x)(A(x)+A(z))\right)\right.\right. \\
& \geq \frac{A(z)}{(A(x)+A(z))^{3}}\left(2 A^{\prime}(x) A(z)+A^{\prime \prime}(x)(A(x)+A(z))\right)
\end{aligned}
$$

The inequality is due to the concavity of $A(x) . A^{\prime \prime}(x) \leq 0$ and $A(0)=0$ implies $A(x) \geq x A^{\prime}(x)$. If the $x$ products have similar demand rates, $A(x)$ is linear. $A^{\prime \prime}(x)=0$ and the cost function is convex in $x$. With strictly concave $A(x)$, the cost function is still convex if $A(x)$ is not too concave. For example, if $A(x)=\ln x$, the sign of the second derivative is determined by $A(z)(-1+2 x)-\ln x$, which is strictly positive for $z \geq e$ and $x>1$.

\section{B Sum of two Gumbel distributed random variables}

The sum of two Gumbel distributions does not have a closed form distribution function. We approximated the sum of Gumbel distributed random variables with a Gumbel distribution. Here we discuss the quality of this approximation. One measure of similarity of two probability distributions is the Kolmogorov-Smirnov (K-S) distance, which is the supremum of the distance between the cumulative density functions. Consider the case with three Gumbel random variables, $X, Y$ and $Z$ with parameters $\left(\eta_{X}, \mu_{X}\right),\left(\eta_{Y}, \mu_{Y}\right)$, and $\left(\eta_{Z}, \mu_{Z}\right)$. The mean of $X+Y$ is $\eta_{X}+\eta_{Y}+\gamma\left(\mu_{X}+\mu_{Y}\right)$

and its variance is $\pi^{2} \mu^{2} / 6$. We set the parameters of $Z$ to have the same mean and variance with $X+Y$ as follows.

$$
\mu_{Z}=\sqrt{\mu_{X}^{2}+\mu_{Y}^{2}} \text { and } \eta_{Z}=\eta_{X}+\eta_{Y}+\gamma\left(\mu_{X}+\mu_{Y}-\mu_{Z}\right)
$$

$\mathrm{K}-\mathrm{S}$ distance is

$$
\sup _{u}\left|F_{X+Y}(u)-F_{Z}(u)\right| \approx \max _{p \in\{0.01, . ., 0.99\}}\left|F_{X+Y}\left(F_{Z}^{-1}(p)\right)-p\right|
$$

We computed $F_{X+Y}$ using numerical integration and searched for the maximum over percentiles for the combination of the following parameters: $\eta_{X}=\{10,40\}, \eta_{Y}=\{10,40\}, \mu_{X}=\{5,20\}$, 
$\mu_{Y}=\{5,20\}$. The K-S distance never exceeded 0.019 in these examples, which we believe is fairly small. It is also of interest to note that the K-S distance seems to be effected by only the ratio of $\mu_{X} / \mu_{Y}$ but none of the other parameters, reaching the maximum at $\mu_{X} / \mu_{Y}=1$. Figure 5 provides an example of the cumulative density functions of $X+Y$ and $Z$.

Finally, from the perspective of our model, we are interested more in how the resulting choice probabilities are affected with the approximation. Consider that $X, Y, A, B$ are all Gumbel distributions, $X+Y$ and $A+B$ are approximated by Gumbels $Z$ and $C$ respectively. We compare $\operatorname{Pr}\{X+Y<A+B\}$ and $\operatorname{Pr}\{Z<C\}$. Notice that the latter one can be computed using the MNL formula. For the combination of the set of parameters above, the maximum difference in probabilities is 0.010 , which is also fairly small and in the same order of magnitude with the K-S distance. Table 4 presents a set of resulting choice probabilities with both the true distributions and the Gumbel approximations.

\section{Derivatives}

Let $\tau=1 / \sqrt{B_{t}}, \Phi=A_{t}(\mathbf{x})+A_{t}(\mathbf{z})$ and $\Delta=A_{t}(\mathbf{z})-A_{t}(\mathbf{x})$. The first and second derivatives of the attractiveness function are

$$
\begin{gathered}
\frac{\partial A_{t}(\mathbf{x})}{\partial x_{j}}=I_{\left\{j \in B_{t}\right\}} \tau A_{t}(\mathbf{x}) A_{j}\left(x_{j}\right)^{-1} A_{j}^{\prime}\left(x_{j}\right) \\
\frac{\partial^{2} A_{t}(\mathbf{x})}{\partial x_{j}^{2}}=I_{\left\{j \in B_{t}\right\}} \tau A_{t}(\mathbf{x}) A_{j}\left(x_{j}\right)^{-1}\left(A_{j}\left(x_{j}\right)^{-1} A_{j}^{\prime}\left(x_{j}\right)^{2}(\tau-1)+A_{j}^{\prime \prime}\left(x_{j}\right)\right) \\
\frac{\partial^{2} A_{t}(\mathbf{x})}{\partial x_{j} \partial x_{k}}=I_{\left\{j, k \in B_{t}\right\}} \tau^{2} A_{t}(\mathbf{x}) A_{j}\left(x_{j}\right)^{-1} A_{k}\left(x_{k}\right)^{-1} A_{j}^{\prime}\left(x_{j}\right) A_{k}^{\prime}\left(x_{k}\right)
\end{gathered}
$$

The first and second derivatives of the share function are derived below.

$$
\frac{\partial s_{t}(\mathbf{x})}{\partial x_{j}}=I_{\left\{j \in B_{t}\right\}} \frac{A_{t}(\mathbf{z})}{\left(A_{t}(\mathbf{x})+A_{t}(\mathbf{z})\right)^{2}} \frac{\partial A_{t}(\mathbf{x})}{\partial x_{j}}=I_{\left\{j \in B_{t}\right\}} \frac{A_{t}(\mathbf{z})}{\Phi^{2}} \tau A_{t}(\mathbf{x}) A_{j}\left(x_{j}\right)^{-1} A_{j}^{\prime}\left(x_{j}\right)
$$




$$
\begin{aligned}
& \frac{\partial^{2} s_{t}(\mathbf{x})}{\partial x_{j}^{2}}=\frac{A_{t}(\mathbf{z})}{\left(A_{t}(\mathbf{x})+A_{t}(\mathbf{z})\right)^{3}}\left(-2\left(\frac{\partial A_{t}(\mathbf{x})}{\partial x_{j}}\right)^{2}+\left(A_{t}(\mathbf{x})+A_{t}(\mathbf{z})\right) \frac{\partial^{2} A_{t}(\mathbf{x})}{\partial x_{j}^{2}}\right) \\
& =I_{\left\{j \in B_{t}\right\}} \frac{A_{t}(\mathbf{z})}{\Phi^{3}}\left(\begin{array}{c}
-2\left(A_{t}(\mathbf{x}) A_{j}\left(x_{j}\right)^{-1} A_{j}^{\prime}\left(x_{j}\right) \tau\right)^{2} \\
+\Phi \tau A_{t}(\mathbf{x}) A_{j}\left(x_{j}\right)^{-1}\left(A_{j}\left(x_{j}\right)^{-1} A_{j}^{\prime}\left(x_{j}\right)^{2}(\tau-1)+A_{j}^{\prime \prime}\left(x_{j}\right)\right)
\end{array}\right) \\
& =I_{\left\{j \in B_{t}\right\}} \frac{A_{t}(\mathbf{z}) A_{t}(\mathbf{x}) A_{j}\left(x_{j}\right)^{-1}}{\Phi^{3}}\left(-2 A_{t}(\mathbf{x}) A_{j}\left(x_{j}\right)^{-1} A_{j}^{\prime}\left(x_{j}\right)^{2} \tau+\Phi\left(\left(A_{j}\left(x_{j}\right)^{-1} A_{j}^{\prime}\left(x_{j}\right)^{2}(\tau-1)+A_{j}^{\prime \prime}\left(x_{j}\right)\right)\right)\right) \\
& =I_{\left\{j \in B_{t}\right\}} \frac{A_{t}(\mathbf{z}) A_{t}(\mathbf{x}) A_{j}\left(x_{j}\right)^{-1} \tau}{\Phi^{3}}\left(A_{j}\left(x_{j}\right)^{-1} A_{j}^{\prime}\left(x_{j}\right)^{2}\left(-2 \tau A_{t}(\mathbf{x})+(-1+\tau) \Phi\right)+\Phi A_{j}^{\prime \prime}\left(x_{j}\right)\right) \\
& =-I_{\left\{j \in B_{t}\right\}} \frac{A_{t}(\mathbf{z}) A_{t}(\mathbf{x}) A_{j}\left(x_{j}\right)^{-1} \tau}{\left(A_{t}(\mathbf{x})+A_{t}(\mathbf{z})\right)^{3}}\left(A_{j}\left(x_{j}\right)^{-1} A_{j}^{\prime}\left(x_{j}\right)^{2}(\Phi-\tau \Delta)-\Phi A_{j}^{\prime \prime}\left(x_{j}\right)\right) \\
& \leq 0 \text {. }
\end{aligned}
$$

$$
\begin{aligned}
\frac{\partial^{2} s_{t}(\mathbf{x})}{\partial x_{k} \partial x_{j}} & =\frac{A_{t}(\mathbf{z})}{\left(A_{t}(\mathbf{x})+A_{t}(\mathbf{z})\right)^{3}}\left(-2 \frac{\partial A_{t}(\mathbf{x})}{\partial x_{k}} \frac{\partial A_{t}(\mathbf{x})}{\partial x_{j}}+\left(A_{t}(\mathbf{x})+A_{t}(\mathbf{z})\right) \frac{\partial^{2} A_{t}(\mathbf{x})}{\partial x_{k} \partial x_{j}}\right) \\
& =I_{\left\{j, k \in B_{t}\right\}} \frac{A_{t}(\mathbf{z})}{\Phi^{3}} \tau^{2} A_{t}(\mathbf{x}) A_{j}\left(x_{j}\right)^{-1} A_{k}\left(x_{k}\right)^{-1} A_{j}^{\prime}\left(x_{j}\right) A_{k}^{\prime}\left(x_{k}\right)\left(A_{t}(\mathbf{z})-A_{t}(\mathbf{x})\right)
\end{aligned}
$$

\section{Statement and proof of intermediate step}

Proposition 11 Consider the special case A. i) If CM symmetric equilibrium $\mathbf{x}$ satisfies $A_{t}(\mathbf{x})>$ $\frac{(2 \tau-1)}{(2 \tau+1)} A_{t}(\mathbf{z})$, for all $t$ where $\tau=1 / \sqrt{\left|B_{t}\right|}$, then $\mathbf{x}$ can not be part of a continuum of equilibria. ii) The game CM has at most one symmetric equilibrium in $X^{-}$. iii) If categories are symmetric and there exists an equilibrium in $X^{-}$, then it is the unique equilibrium in $X^{-}$and it is symmetric.

Proof. $i$ ) As we mentioned before, the game (CM) has multiple equilibria, but we use the uniqueness conditions as an argument in our proof. Showing that a best reply map $\left(x_{j}\left(x_{-j}\right)\right)_{j=1, . ., N}$ is a contraction mapping is sufficient for a game to have a unique equilibrium. A sufficient condition for a mapping to be a contraction is that the Jacobian of $\pi_{x_{j}}^{j}=0, j=1 . . N$ is diagonally dominant (Section 2.5., Vives 1999), i.e.,

$$
\begin{array}{r}
\pi_{x_{j} x_{j}}^{j}(\mathbf{x})+\sum_{k \neq j}\left|\pi_{x_{j} x_{k}}^{j}(\mathbf{x})\right|<0 \\
\sum_{t \mid j \in B_{t}} p_{j} \lambda_{t} \frac{\partial^{2} s_{t}(\mathbf{x})}{\partial x_{j}^{2}}-C_{j}^{\prime \prime}\left(x_{j}\right)+\sum_{k \neq j} \sum_{t \mid\{j, k\} \subseteq B_{t}} p_{j} \lambda_{t}\left|\frac{\partial^{2} s_{t}(\mathbf{x})}{\partial x_{k} \partial x_{j}}\right|<0
\end{array}
$$

In the special case $\mathrm{A}, \cap_{k \neq j}\left\{t \mid j, k \in B_{t}\right\}=\varnothing$, and $\cup_{k \neq j}\left\{t \mid j, k \in B_{t}\right\} \subseteq\left\{t \mid j \in B_{t}\right\}$. So there is no baskets counted twice in the double summation. Hence, a sufficient condition for diagonal 
dominance is to show that $\left|\frac{\partial^{2} s_{t}(\mathbf{x})}{\partial x_{k} \partial x_{j}}\right|<-\frac{\partial^{2} s_{t}(\mathbf{x})}{\partial x_{j}^{2}}$ for all $t, j$, and $k$. From (10) and (11), we see that sufficient condition $\left|\frac{\partial^{2} s_{t}(\mathbf{x})}{\partial x_{k} \partial x_{j}}\right|<-\frac{\partial^{2} s_{t}(\mathbf{x})}{\partial x_{j}^{2}}, \forall t$ may not hold in general. However, it holds when $A_{j}\left(x_{j}\right)=A_{k}\left(x_{k}\right), A_{j}^{\prime}\left(x_{j}\right)=A_{k}^{\prime}\left(x_{k}\right)$, and $(\Phi-\tau \Delta)>\tau|\Delta|$. In $X^{+}$, the last condition is equivalent to $A_{t}(\mathbf{x})>\frac{(2 \tau-1)}{(2 \tau+1)} A_{t}(\mathbf{z}) \quad$ (the coefficient $\frac{(2 \tau-1)}{(2 \tau+1)}$ is approximately 0.17 . Therefore the proposition applies to a large part of the strategy space. In $X^{-},|\Delta|=-\Delta,(\Phi-\tau \Delta)>-\tau \Delta$ is satisfied. Notice that the sufficiency condition for diagonal dominance is achieved without using the nonnegative terms $-\Phi A_{j}^{\prime \prime}\left(x_{j}\right)$ and $\frac{\partial^{2} s_{t}(\mathbf{x})}{\partial x_{j}^{2}}$ for $t: j \in B_{t}, k \notin B_{t}$ in the argument. Due to the slacks not used in proving the sufficiency condition and the continuity of all the terms, we can argue (or formally prove) that diagonal dominance of the Jacobian is satisfied not only at the diagonal but also in an $\varepsilon$ neighborhood of the diagonal when $\varepsilon$ is chosen sufficiently small.

Using this result, we define new games on smaller compact $N$-cubes around the diagonal of $\Re^{N}$. Since the best response map is a contraction there is a unique equilibrium in that cube. The implication of this result on the original game $\mathrm{CM}$ is that there cannot be a continuum of equilibria or equilibria too close to each other around the diagonal.

ii) Since all the best response functions $x_{j}\left(x_{-j}\right)$ are decreasing in all components of $x_{-j}$ in $X^{-}$, if there is a symmetric equilibrium in $X^{-}$, there can not be a larger symmetric equilibrium. Therefore, there can be at most one symmetric equilibrium in the submodular region of CM.

iii) When categories are symmetric, since the reaction functions are decreasing, the only possibility to have multiple equilibria in $X^{-}$is when there is a continuum of equilibria around the symmetric equilibrium, (e.g., reaction functions with a slope of -1). But this is ruled out by part $(i)$.

\section{References}

[1] ACNielsen. 1998. Eighth Annual Survey of Trade Promotion Practices. Chicago, IL. ACNielsen.

[2] Agrawal N., S.A. Smith. 2003. Optimal retail assortments for substitutable items purchased in sets. Naval Research Logistics. 50 (7) 793-822.

[3] Anderson, S.P., A. de Palma., J.F. Thisse. 1992. Discrete choice theory of product differentiation. Cambridge, MA. The MIT Press.

[4] Basuroy, S., M.K. Mantrala, R.G. Walters. 2001. The impact of category management on retailer prices and performance: Theory and evidence. Journal of Marketing. 65 16-32. 
[5] Basuroy, S., D. Nguyen. 1998. Multinomial logit market share models: Equilibrium characteristics and strategic implications. Management Science. 44 (10) 1396-1408.

[6] Bell, D. R., T-H Ho, C.S. Tang 1998. Determining where to shop: Fixed and variable costs of shopping. Journal of Marketing Research. 35 352-369.

[7] Bell, D.R., J.M. Lattin. 1998. Shopping behavior and consumer preference for store price format: Why large basket shoppers prefer EDLP. Marketing Science. 17 66-88.

[8] Ben-Akiva, M. S.R. Lerman. 1985. Discrete choice analysis: Theory and application to travel demand. Cambridge, MA. The MIT Press.

[9] Boatwright, P. J.C. Nunes. 2001. Reducing assortment: An attribute-based approach. Journal of Marketing. 65 (3) 50-63.

[10] Bodapati, A.V., V. Srinivasan. 1999. The impact of out-of-store advertising on store sales. Working paper. Nortwestern University.

[11] Broniarczyk, S.M., W.D. Hoyer, L. McAlister. 1998. Consumers' perception of the assortment offered in a grocery category: The impact of item reduction. J. of Mrkt Res. 35 166-76.

[12] Bultez, A., P. Naert. 1988. SHARP: Shelf allocation for retailers profit. Marketing Science. 7 211-231.

[13] Cattani, K., E.Dahan, G.Schmidt. 2003. Spackling: Smoothing make-to-order production of custom products with make-to-stock productions of standard items. Working paper, UNC-CH.

[14] Chen, Y., J.D. Hess, R.T. Wilcox, Z.J. Zhang. 1999. Accounting profits versus marketing profits: A relevant metric for category management. Marketing Science. 18 (3). 208-229.

[15] Dhar, S.K., S.J. Hoch, N. Kumar. 2001. Effective category management depends on the role of the category. Journal of Retailing. 77 (2) 165-184.

[16] Dreze, X., S.J. Hoch, M.E. Purk. 1994. Shelf management and space elasticity. Journal of Retailing. 70 301-26.

[17] Fudenberg,D., J. Tirole 2000. Game Theory. Cambridge, MA. The MIT Press.

[18] Gruca, T.S., D. Sudharshan. 1991. Equilibrium characteristics of multinomial logit market share models. Journal of Marketing Research. 28 (11) 480-482.

[19] Guadagni, P.M., J.D.C. Little. 1983. A logit model of brand choice calibrated on scanner data. Marketing Science. 2 203-238.

[20] Hoch, S.J., E.T. Bradlow, B. Wansink. 1999. The variety of an assortment. Mrkt Sci. 25 342-55.

[21] Horn, R.A., C.R. Johnson. 1996. Matrix Analysis. NY, NY. Cambridge University Press. 
[22] Huffman, C., B.E. Kahn. 1998. Variety for sale: Mass customization or mass confusion? Journal of Retailing $\mathbf{7 4}$ 491-513.

[23] Kahn, B.E., D.R.Lehmann. 1991. Modeling choice among assortment, J of Ret. 67 274-275.

[24] Karnani, A. 1985. Strategic implicationd of market share attraction models. Mgmt Sci. 31 (5) 536-547.

[25] Kok, A.G., M.L. Fisher. 2004. Demand estimation and assortment optimization under substitution: Methodology and application. Working paper, Fuqua School of Business.

[26] Kurt Salmon Associates. 1993. Efficient consumer response: Enhancing consumer value in the grocery industry. Food Marketing Institute Report \# 9-526. Food Marketing Institute.

[27] Mahajan, S., G. van Ryzin. 2001. Stocking retail assortments under dynamic consumer substitution. Operations Research. 49 334-351.

[28] Manchanda, P., A. Ansari, S. Gupta. 1999. The "shopping basket": A model for multicategory purchase incidence decisions. Marketing Science. 18 (2). 95-114.

[29] McGillivray, A.R., E.A. Silver. 1978. Some concepts for inventory control under subsitutable demand. INFOR. 16. 47-63.

[30] Monahan, G.E. 1987. The structure of equilibria in market share attraction models. Management Science. 33 (2) 228-243.

[31] Netessine, S., N. Rudi. 2003. Centralized and competitive inventory models with demand substitution. Operations Research. 51. 329-335.

[32] Netessine S., F. Zhang. 2003. The impact of supply-side externalities among downstream firms on supply chain efficiency. Working paper. The Wharton School. University of Pennsylvania.

[33] Quelch, J.A., D. Kenny. 1994. Extend profits, not product lines. Harvard Bus Rev. 72 153-160.

[34] Parlar, M. 1988. Game theoretic analysis of the substitutable product inventory problem with random demands. Naval Research Logistics. 35. 397-409.

[35] Progressive Grocer. 1996. Can retailer-controlled brands dominate category management? 75. A9-A10.

[36] Rhee, H., D.R. Bell. 2002. The inter-store mobility of supermarket shoppers. J of Ret. 78 (4) $225-237$.

[37] Russell, G.J., D.R. Bell, et al. 1997. Perspectives on multiple category choice. Marketting Letters. 8 (3) 297-305.

[38] Smith, S.A., N. Agrawal. 2000. Management of multi-item retail inventory systems with demand substitution. Operations Research. 48 50-64. 
[39] Simonson, I. 1999. The effect of product assortment on buyer preferences. J of Ret. 75 347-70.

[40] Topkis D.M. 1998. Supermodularity and Complementarity. Princeton University Press..

[41] van Ryzin, G., S. Mahajan. 1999. On the relationship between inventory costs and variety benefits in retail assortments. Management Science. 45 1496-1509.

[42] Vives, X. 1999. Oligopoly pricing: old ideas and new tools. Cambridge, MA. The MIT Press.

[43] Zenor, M.J. 1994. The Profit benefits of category management. J of Mrkt Res. 31 (2). 202-213. 


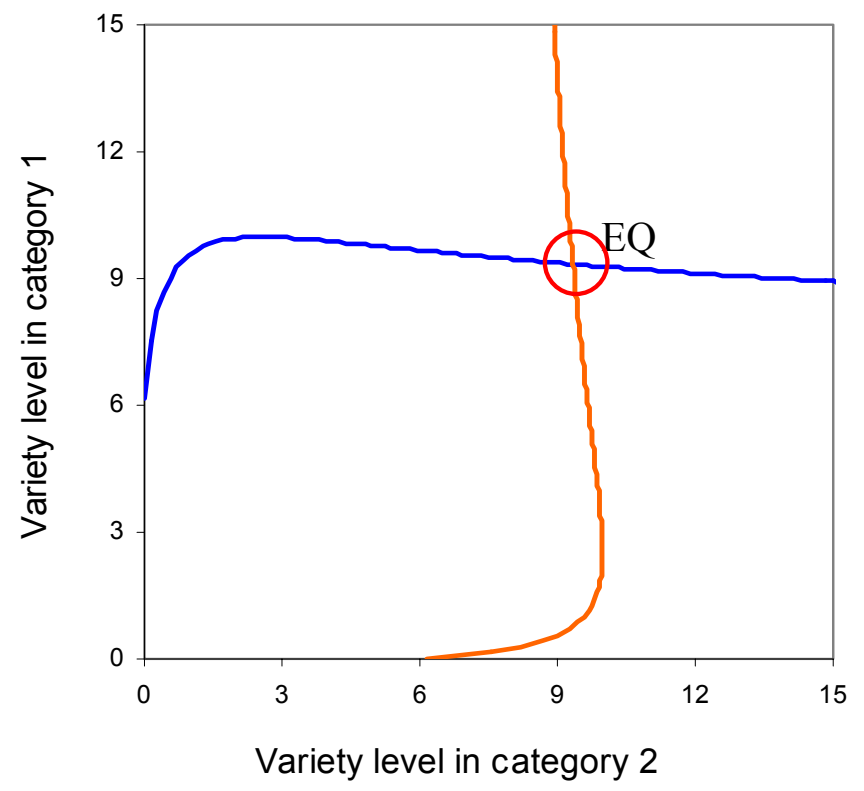

Figure 1. A two category example with unique category management equilibrium. $\mathrm{N}=2$, symmetric categories, $\mathrm{p}_{\mathrm{j}}=1, \mathrm{~A}_{\mathrm{j}}(\mathrm{x})=\mathrm{x}, \mathrm{C}_{\mathrm{j}}(\mathrm{x})=\mathrm{c}_{\mathrm{j}} \mathrm{x}, \mathrm{c}_{\mathrm{j}}=2, \mathrm{z}_{\mathrm{j}}=5$ for all $j$. $\lambda_{1}=50, \lambda_{2}=50, \lambda_{12}=50$.

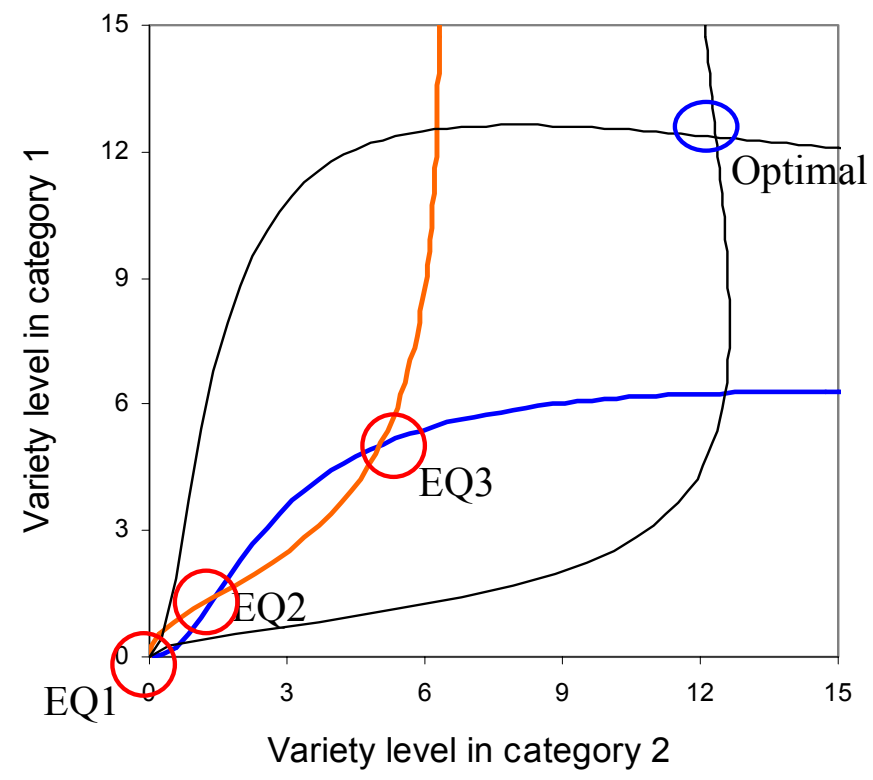

Figure 2. A two category example depicting the three category management equilibria and the optimal solution. $\mathrm{N}=2$, symmetric categories, $\mathrm{p}_{\mathrm{j}}=1$, $\mathrm{A}_{\mathrm{j}}(\mathrm{x})=\mathrm{x}, \mathrm{C}_{\mathrm{j}}(\mathrm{x})=\mathrm{c}_{\mathrm{j}} \mathrm{x}, \mathrm{c}_{\mathrm{j}}=2.8, \mathrm{z}_{\mathrm{j}}=5$ for all $j . \lambda_{1}=0, \lambda_{2}=0, \lambda_{12}=100$. 


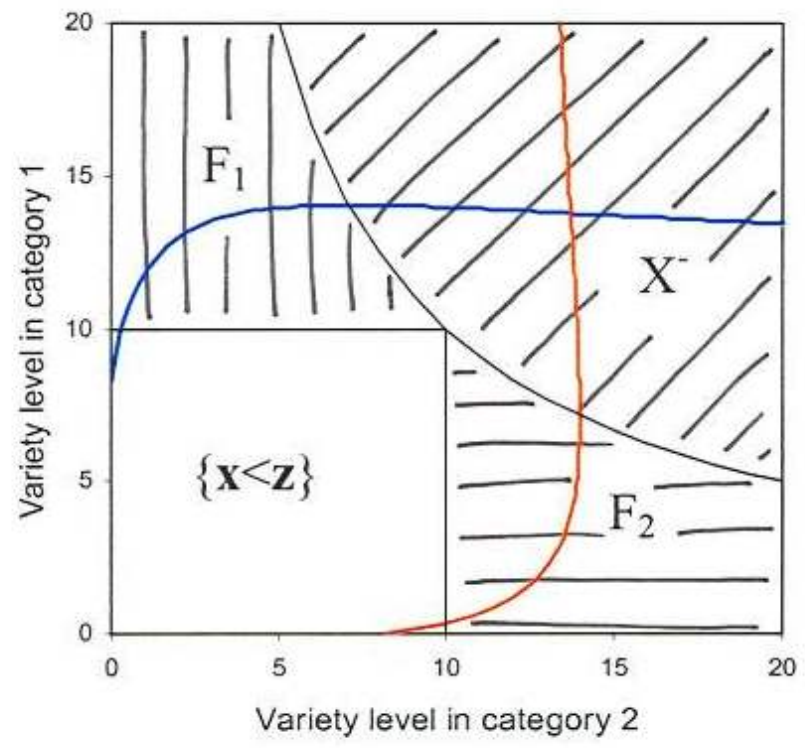

Figure 3. A two category example illustrating the regions relevant to the proof of Proposition 5. N=2, symmetric categories, $\mathrm{p}_{\mathrm{j}}=1, \mathrm{~A}_{\mathrm{j}}(\mathrm{x})=\mathrm{x}, \mathrm{C}_{\mathrm{j}}(\mathrm{x})=\mathrm{c}_{\mathrm{j}} \mathrm{x}, \mathrm{c}_{\mathrm{j}}=3$, $\mathrm{z}_{\mathrm{j}}=10$ for all $j \cdot \lambda_{1}=100, \lambda_{2}=100, \lambda_{12}=100$.

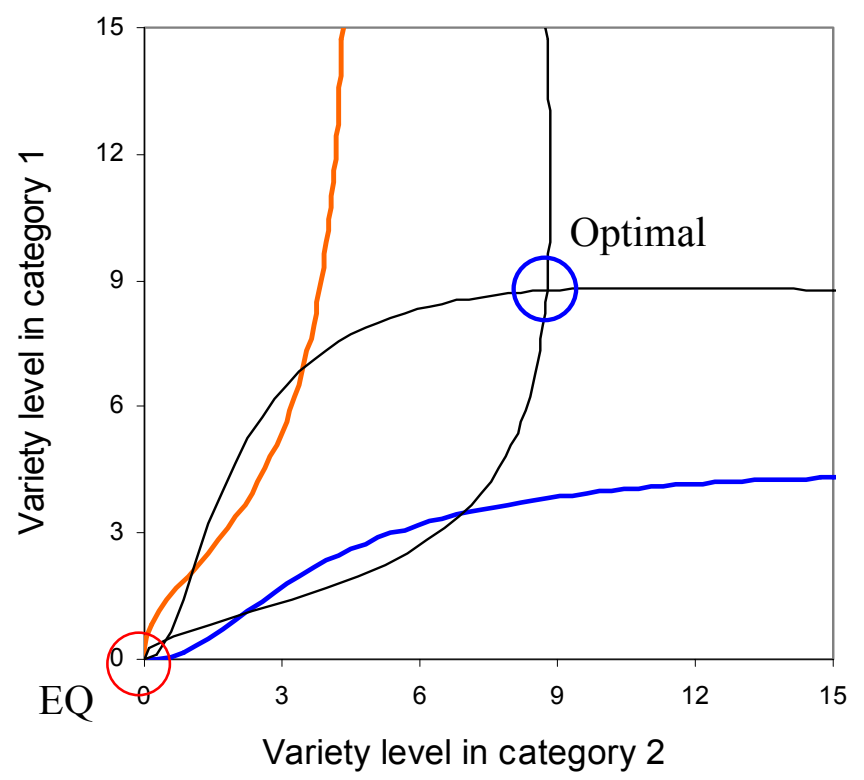

Figure 4. A two category example depicting the unique category management equilibrium at zero and the optimal solution. $\mathrm{N}=2$, symmetric categories, $\mathrm{p}_{\mathrm{j}}=1, \mathrm{~A}_{\mathrm{j}}(\mathrm{x})=\mathrm{x}, \mathrm{C}_{\mathrm{j}}(\mathrm{x})=\mathrm{c}_{\mathrm{j}} \mathrm{x}, \mathrm{c}_{\mathrm{j}}=4, \mathrm{z}_{\mathrm{j}}=10$ for all $j . \lambda_{1}=0, \lambda_{2}=0, \lambda_{12}=100$. 


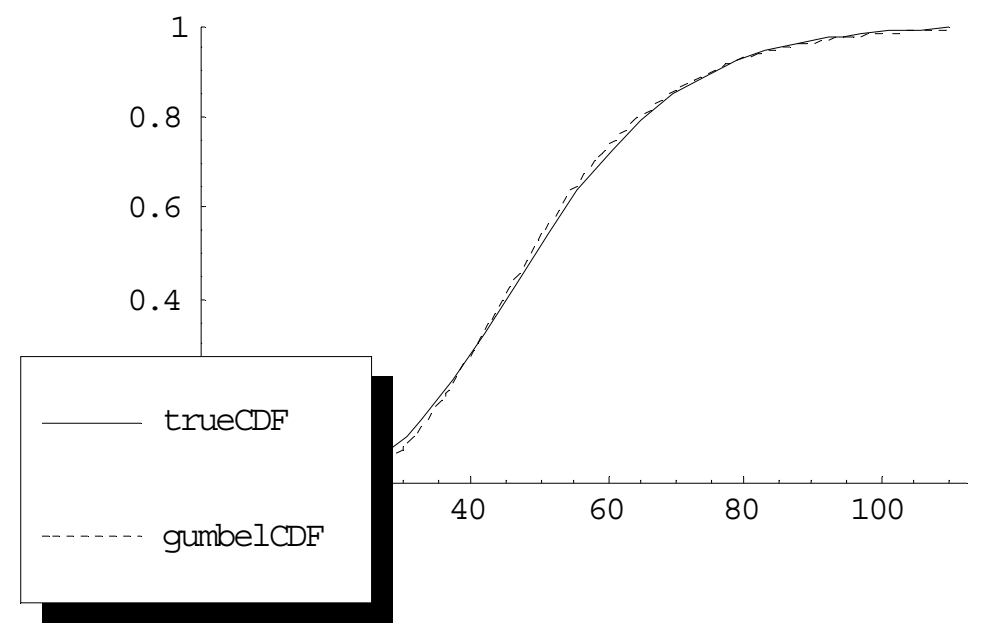

Figure 5. Cumulative density functions of the sum of two Gumbel random variables and its Gumbel approximation. $\eta_{x}=\eta_{y}=20, \mu_{x}=\mu_{y}=10$.

Table 1. Allocation of basket shoppers in numerical experiments. First entry in a vector is the percentage of shoppers with a basket of size one, the second entry is the shoppers with a basket size of two, and so on.

\begin{tabular}{|c|c|c|c|}
\hline Proportion of basket shoppers & $N=2$ & $N=3$ & $N=5$ \\
\hline low & $(.8, .2)$ & $\begin{array}{l}(.8, .2,0) \\
(.5, .5,0)\end{array}$ & $\begin{array}{l}(.8, .2,0,0,0) \\
(.5, .5,0,0,0) \\
(.6, .2, .2,0,0)\end{array}$ \\
\hline medium & $(.5, .5)$ & $\begin{array}{l}(.2, .8,0) \\
(.6, .2, .2)\end{array}$ & $\begin{array}{l}(.2, .6, .2,0,0) \\
(.2, .2, .6,0,0) \\
(.2, .2, .2, .2, .2)\end{array}$ \\
\hline high & $(.2, .8)$ & $\begin{array}{c}(.2, .2, .6) \\
(0, .2, .8)\end{array}$ & $\begin{array}{l}(.2,0, .6,0, .2) \\
(.2,0, .2,0, .6) \\
(0,0, .2, .2, .6)\end{array}$ \\
\hline
\end{tabular}


Table 2. Summary of numerical results.

\begin{tabular}{|c|c|c|c|}
\hline & & $\begin{array}{l}\text { Profit loss, } \\
1-\Pi^{\mathrm{cm}} / \Pi^{\circ}\end{array}$ & $\begin{array}{c}\text { Decrease in variety } \\
\text { level, } 1-x^{c m} / x^{0}\end{array}$ \\
\hline \multirow{3}{*}{$\mathrm{N}$} & 2 & $13 \%$ & $31 \%$ \\
\hline & 3 & $23 \%$ & $41 \%$ \\
\hline & 5 & $35 \%$ & $51 \%$ \\
\hline \multirow{2}{*}{$z$} & low & $14 \%$ & $34 \%$ \\
\hline & high & $41 \%$ & $55 \%$ \\
\hline \multirow{2}{*}{ C } & low & $14 \%$ & $34 \%$ \\
\hline & high & $41 \%$ & $55 \%$ \\
\hline Proportion & low & $4 \%$ & $20 \%$ \\
\hline of basket & medium & $27 \%$ & $47 \%$ \\
\hline shoppers & high & $51 \%$ & $66 \%$ \\
\hline
\end{tabular}

Table 3. Performance of the category management (CM) and the basket profits heuristic (CM_B) in comparison to the optimal solution. (B and $\mathrm{W}$ denotes the best and worst equilibria of CM_B, respectively).

\begin{tabular}{|c|c|c|c|c|c|c|c|}
\hline \multirow[b]{2}{*}{$\mathrm{N}$} & \multirow[b]{2}{*}{ Data } & \multicolumn{3}{|c|}{ Average profit loss } & \multicolumn{3}{|c|}{ Average deviation in variety levels } \\
\hline & & $1-\Pi^{\mathrm{cm}} / \Pi^{\circ}$ & $1-\Pi^{\mathrm{B}} / \Pi^{\mathrm{o}}$ & $1-\Pi^{\mathrm{w}} / \Pi^{\mathrm{o}}$ & $1-x^{c m} / x^{0}$ & $1-x^{B} / x^{O}$ & $1-x^{W} / x^{0}$ \\
\hline \multirow{2}{*}{2} & Symmetric & $13.4 \%$ & $0.2 \%$ & $8.5 \%$ & $30.6 \%$ & $-4.7 \%$ & $4.0 \%$ \\
\hline & Asymmetric & $6.8 \%$ & $0.1 \%$ & $0.1 \%$ & $26.5 \%$ & $-3.9 \%$ & $-3.7 \%$ \\
\hline$\overline{3}$ & Symmetric & $22.8 \%$ & $0.3 \%$ & $21.1 \%$ & $40.8 \%$ & $-5.7 \%$ & $15.5 \%$ \\
\hline \multicolumn{2}{|c|}{ Average } & $13.2 \%$ & $0.2 \%$ & $8.5 \%$ & $31.8 \%$ & $-4.5 \%$ & $4.0 \%$ \\
\hline
\end{tabular}

Table 4. Resulting choice probabilities using the true distribution of the sum of two Gumbel random variables and its multinomial logit approximation.

\begin{tabular}{ccccc}
\multicolumn{2}{c}{ Mean } & & \multicolumn{2}{c}{$\operatorname{Pr}\{\mathrm{X}+\mathrm{Y}>\mathrm{A}+\mathrm{B}\}$} \\
$(\mathrm{X}, \mathrm{Y})$ & $(\mathrm{A}, \mathrm{B})$ & & TRUE & $\mathrm{MNL}$ \\
\cline { 1 - 2 }$(5,5)$ & $(10,10)$ & & 0.401 & 0.395 \\
$(5,10)$ & $(10,10)$ & & 0.458 & 0.456 \\
$(10,10)$ & $(10,10)$ & & 0.500 & 0.500 \\
$(5,20)$ & $(10,10)$ & & 0.542 & 0.544 \\
$(20,20)$ & $(10,10)$ & & 0.661 & 0.669 \\
\hline
\end{tabular}

